\title{
Theory of borehole stability when drilling through salt formations
}

\author{
José M. Carcione ${ }^{1}$, Hans B. Helle ${ }^{2}$, and Anthony F. Gangi ${ }^{3}$
}

\begin{abstract}
We present a mathematical analysis of borehole stability when drilling through rock salt. First, we consider an elastic transversely isotropic medium and find the optimal mud weight as a function of the vertical overburden and horizontal tectonic stresses. Then, the Zener and Maxwell mechanical models are used to model the effects of transient and steady-state creep flow, respectively, in isotropic media. Under certain conditions such as the absence of dilatational anelasticity, the Burger model can be used to describe the steady-state flow, including transient creep effects. The type of creep is regulated by critical octahedral-stress values that depend on temperature and pressure. A typical drilling results in conditions of plane strain, whose solution is given by Kirsch's equations. In this case, the borehole is subject to minimum and maximum horizontal stresses, which differ from the vertical stress. The analysis provides expressions for the shape of the borehole-cross section, the boreholewall closure time, and the optimal mud weight to avoid wall collapse or expansion. It is shown that an anisotropic state of tectonic stress may require mud pressures exceeding the overburden stress and that the calculation should consider the joint optimization of the shape and area of the borehole cross section.
\end{abstract}

\section{INTRODUCTION}

Exploration areas where massive salt bodies are dominating features constitute a challenge for drilling operations (Bradley, 1978; Infante and Chenevert, 1986; Kim, 1988, Leblanc, 1994a, b, c). Depending on well conditions (tectonic stress, temperature, and mud weight), salt shows transient and steady-state creep behavior, which may cause hole closure or lost circulation. Rock salt be- haves differently from other rocks in that it has the ability to creep and flow significantly with time, depending on the stress and temperature conditions. Creep and flow lead to borehole deformations which may cause complications such as stuck pipe and casing collapse (after drilling and cementation, salt creep and subsequent casing collapse may occur). It is therefore clear that significant benefits can be obtained from quantifying the magnitude and timing of salt loading (Willson, 2003).

Rock salt mainly flows when subjected to distortional or deviatoric stress. Under isotropic stress (often called hydrostatic stress), rock salt does not flow appreciably even though it will deform elastically (small strains or deformations). It flows under isotropic stress to achieve (minor) porosity reduction. Because the deformation is small for isotropic stresses, the major flow deformation is associated with the deviatoric stress. The magnitude of the deviatoric stress is proportional to the octahedral stress (see below), a scalar that is invariant under coordinate transformations and whose value determines the character of the flow of the rock salt around the borehole.

Our objective is to present a theoretical study of the problem of stability of a borehole drilled through a salt formation with the purpose of evaluating the optimal mud weight and avoiding creep effects. Early theoretical studies on borehole stability were based on models developed for underground salt cavities (e.g., Serata and Gloyna, 1960). Infante and Chenevert (1986) use an elastic rheology to obtain the optimal mud weight for boreholes. Their analysis does not consider creep flow and assumes that the medium is isotropic and that the (horizontal) tectonic stress is also isotropic and equal to the overburden stress. Under these conditions, the solution has azimuthal symmetry around the borehole axis.

We do not consider poroelastic and chemical effects, although the former can be important in some situations when pore pressure induced by anisotropy in rock properties and stress distribution become significant (Abouleisman et al., 1996; Abouleisman and Cui, 1998). However, the reality is that porosity effects are small and negligible in most rock salt formations, at least where data are

Manuscript received by the Editor October 22, 2004; revised manuscript received June 15, 2005; published online May 26, 2006.

${ }^{1}$ Instituto Nazionale di Occeanografia e di Geofisica Sperimentale (OGS), Borgo Grotta Gigante 42c, 34010 Sgonico, Trieste, Italy. E-mail: jcarcione @ ogs.trieste.it.

${ }^{2}$ Formerly Norsk Hydro a.s., Oil and Energy Research Center; presently Nyborg Geoconsult, Heiane 103, N-5131 Nyborg, Norway. E-mail: hbhelle @ broadpark.no.

${ }^{3}$ Texas A\&M University, Department of Geology and Geophysics, College Station, Texas 77843-3114. E-mail gangi@tamu.edu. (C) 2006 Society of Exploration Geophysicists. All rights reserved. 
available, implying that stress induced by fluid invasion from the well into the formation can be neglected. The extremely low permeability of salt clearly demonstrates that. Salt is even less permeable than the most impermeable shales, which have permeabilities less than nano Darcies. Hence, poroelastic effects are not important for salt, at least for the initial behavior. The porosity of the salt (crack porosity when the salt goes to failure) may become important when the rock salt has extruded large distances and begins to fail, but our focus here is the behavior of the salt during drilling and before casing.

Near failure, permeability may play a role when the diffusion of the pore fluid creates stress-induced poroelastic effects. Ghassemi and Diek (2001) have shown that, although chemical osmosis in shales reduces the pore pressure and stabilizes the borehole, ion diffusion into the formation may induce tensile radial stress. An important extension of the theory is applying the generalization to inclined boreholes. When the principal stresses are not vertical or horizontal, corresponding to the axis of the borehole (and the normal to the formation), the problem becomes truly $3 \mathrm{D}$, because generally, all the shear components are different from zero (e.g., Cui et al; 1997; Ekbote and Abouleisman, 2003; Roegiers, 2002).

In this work, we generalize Infante and Chenevert's approach in several respects. First, we consider a transversely isotropic elastic medium and a cylindrical stress state; that is, one in which there is equal horizontal principal stress but a different vertical principal stress. The solution for this case also has azimuthal symmetry. Second, we assume an isotropic viscoelastic medium and a true triaxial stress state, i.e., the borehole is subject to minimum and maximum horizontal — tectonic — principal stresses, which differ from the vertical — overburden — principal stresses. In this case, the solution does not exhibit azimuthal symmetry and describes transient and steady-state creep flow and elliptical deformations of the borehole cross section.

We consider an unbounded homogeneous layer of rock salt and a borehole of infinite length with an initial circular cross section. Drilling a borehole under constant tectonic-stress conditions is similar to a creep experiment, so the problem is to obtain the corresponding creep functions or time-varying strains. One principalstress direction is vertical, and the problem becomes one of plane strain if the medium has a vertical symmetry axis, i.e., a horizontally layered isotropic medium or a transversely isotropic medium with a vertical symmetry (polar) axis.

There is experimental evidence that linear viscoelastic models are appropriate to describe the behavior of rock salt. Gangi (1981, 1983), among others, obtained exponential functions of time using linear viscoelastic models to fit data for synthetic and natural rock salt. Some of these experiments lasted more than 600 hours (25 days). The viscoelastic creep — with steady-state creep - of salt can be described by a Burger's model which includes the transient creep of the Zener model, which does not exhibit steady-state creep, and the steady-state creep of a Maxwell model. The Burger model, corresponding to the rigidity modulus, is shown in Figure 1 (the Maxwell and Zener model are particular cases of the Burger model as demonstrated in the figure caption). The Zener model is used under pressure and temperature conditions, where the rock behaves as a viscoelastic solid. The choice depends on the value of the elastic octahedral stress, which determines the limit separating transient flow from unrecoverable steady-state flow. When the hole conditions are such that the octahedral stress exceeds a given threshold (the elastic octahedral-stress limit, see below), we use the
Maxwell model, which describes the behavior of a viscoelastic fluid.

The procedure to obtain the optimal mud weight and borehole shrinkage (reduction of the hole radius) is to calculate how the stress state and octahedral stress vary after drilling. The analysis also yields the borehole-section deformation in the case of differential horizontal stress. As mentioned above, the goal is to obtain the time-varying strains or creep functions. For isotropic viscoelastic media, there are two creep functions, related to the two fundamental deformations of the medium, i.e., dilatations and distortions. These basic creep functions correspond to the bulk and shear moduli and viscosities, etc. In the plane-strain (or plane-stress) problem, the strain components depend on combinations of these basic creep functions. The corresponding creep coefficients are calculated using the method of partial fractions in the frequency domain.

\section{MEAN AND OCTAHEDRAL STRESSES}

We use the octahedral-stress theory to describe the deformation of the borehole in the salt formation. In cylindrical coordinates $(r, \theta, z)$, we define the mean stress

$$
\sigma_{m}=\frac{1}{3}\left(\sigma_{r r}+\sigma_{\theta \theta}+\sigma_{z z}\right),
$$

and the octahedral stress

$$
\tau_{o}=\frac{1}{3} \sqrt{\left(\sigma_{r r}-\sigma_{\theta \theta}\right)^{2}+\left(\sigma_{r r}-\sigma_{z z}\right)^{2}+\left(\sigma_{\theta \theta}-\sigma_{z z}\right)^{2}+6\left(\sigma_{r \theta}^{2}+\sigma_{r z}^{2}+\sigma_{\theta z}^{2}\right)},
$$

where $\sigma$ equals the stress components. The borehole problem can be treated as a state of plane strain, where the vertical displacement is constant with radial position, and the horizontal displacements are independent of $z$. In a plane state of strain, the strain components $\epsilon_{r z}=\epsilon_{\theta z}=0$. As a consequence, applying Hooke's law, the stress components $\sigma_{r z}$ and $\sigma_{\theta z}$ vanish, at least for media having

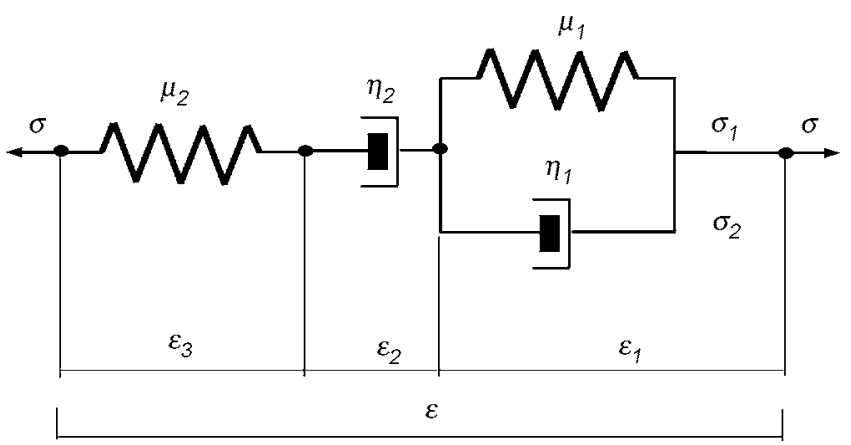

Figure 1. Burger's viscoelastic model for the shear modulus. It is an electric-circuit analog for the mechanical system with stress analogous to current and strain analogous to voltage (or potential). The stress-strain relations of each constituent are: $\sigma_{1}=\mu_{1} \epsilon_{1}, \sigma_{2}$ $=\eta_{1} \dot{\epsilon}_{1}, \sigma=\eta_{2} \dot{\epsilon}_{2}$ and $\sigma=\mu_{2} \epsilon_{3}$. Using the relations $\epsilon=\epsilon_{1}+\epsilon_{2}$ $+\epsilon_{3}$ and $\sigma=\sigma_{1}+\sigma_{2}$, we obtain equation 100 . The Zener model is obtained for $\eta_{2} \rightarrow \infty$ (i.e., when $\dot{\epsilon}_{2} \rightarrow 0$ ); the Kelvin-Voigt model is obtained for $\mu_{2} \rightarrow \infty$ (when $\epsilon_{3} \rightarrow 0$ ) and $\eta_{2} \rightarrow \infty$ (when $\dot{\epsilon}_{2}$ $\rightarrow 0$ ); and the Maxwell model is obtained for $\mu_{1} \rightarrow \infty$ (when $\epsilon_{1}$ $\rightarrow 0)$, and $\eta_{1} \rightarrow \infty\left(\right.$ when $\left.\dot{\epsilon}_{1} \rightarrow 0\right)$. 
orthorhombic and higher symmetries (e.g., Charlez, 1991). The stresses defined in equations 1 and 2 are invariants because they are independent of the choice of reference axis (e.g., Pande et al., 1990).

Before drilling, or at $r \rightarrow \infty$, (far-field stresses), we have

$$
\begin{aligned}
& \sigma_{r r}=\frac{\left(\sigma_{H}+\sigma_{h}\right)}{2}+\frac{\left(\sigma_{H}-\sigma_{h}\right)}{2} \cos 2 \theta, \\
& \sigma_{\theta \theta}=\frac{\left(\sigma_{H}+\sigma_{h}\right)}{2}-\frac{\left(\sigma_{H}-\sigma_{h}\right)}{2} \cos 2 \theta, \\
& \sigma_{r \theta}=-\frac{\left(\sigma_{H}-\sigma_{h}\right)}{2} \sin 2 \theta, \\
& \sigma_{z z}=\sigma_{v} .
\end{aligned}
$$

(e.g., Jaeger and Cook, 1969; Charlez, 1991), where $\sigma_{H}$ and $\sigma_{h}$ are the maximum and minimum horizontal (tectonic) stresses (at $\theta$ $=0$ and $\theta=\pi / 2$, respectively), and the vertical overburden stress, $\sigma_{v}$, can be determined from the density log as

$$
\sigma_{v}=-g \int_{0}^{z} \rho(z) d z
$$

where $\rho$ is the density and $g$ is the acceleration of gravity.

Figure 2 shows the octahedral stress as a function of the octahedral strain. When the stress vector associated with the normal to the octahedral plane is generated, its components in the principal directions are the eigenstresses (or principal stresses). Alternatively, it has two components - one normal to the plane, which has a magnitude equal to the mean stress and one tangential to the plane, which has a magnitude equal to the octahedral stress. The latter is proportional to the magnitude of the deviatoric stress.

The rock starts to yield when $\tau_{o}$ exceeds the elastic octahedralstress limit $\tau_{o e}$. Below this limit, there is gradual creep deformation when constant stress is applied. Then, if $\tau_{o}$ is lower than the elastic limit $\tau_{o e}$, the material follows a viscoelastic stress-strain relation. If $\tau_{o}$ lies between $\tau_{o e}$ and the plastic limit $\tau_{o p}$, steady-state flow occurs. Beyond $\tau_{o p}$ failure is likely to occur.

Before drilling, the mean and octahedral stresses are obtained by substituting equation 3 into equations 1 and 2 . It gives

$$
\sigma_{m}=\frac{1}{3}\left(\sigma_{H}+\sigma_{h}+\sigma_{v}\right)
$$

and

$$
\tau_{o}=\frac{\sqrt{2}}{3} \sqrt{\left(\frac{\sigma_{H}+\sigma_{h}}{2}-\sigma_{v}\right)^{2}+3\left(\frac{\sigma_{H}-\sigma_{h}}{2}\right)^{2}} .
$$

For brevity, we use the following notation in the case of axisymmetry: $\sigma_{r}=\sigma_{r r}, \sigma_{\theta}=\sigma_{\theta \theta}, \sigma_{z}=\sigma_{z z}, \epsilon_{r}=\epsilon_{r r}, \epsilon_{\theta}=\epsilon_{\theta \theta}$, and $\epsilon_{z}=\epsilon_{z z}$.

\section{ELASTIC STRESSES IN ANISOTROPIC ROCK}

There are three main factors to consider rock salt an anisotropic medium. First, rock salt crystals are intrinsically anisotropic with cubic symmetry (Sun et al., 1991). Second, the deformation of salt during flow leads to preferred lattice orientation of the constituent crystals, leading to effective transverse isotropy, according to Raymer et al. (2000). Third, transverse isotropy can be induced by the tectonic stress.

The combination of these three effects may lead to lower symmetries than transverse isotropy, depending on the orientation of the princpal axes related to each cause. In this section, we assume $\sigma_{h}=\sigma_{H}$ and a transversely isotropic medium whose symmetry axis is parallel to the borehole axis, and we generalize the equations given by Serata and Gloyna (1960) and Infante and Chenevert (1986).

The strain-stress relations are

$$
\begin{aligned}
& \epsilon_{r}=s_{11} \sigma_{r}+s_{12} \sigma_{\theta}+s_{13} \sigma_{z}, \\
& \epsilon_{\theta}=s_{12} \sigma_{r}+s_{11} \sigma_{\theta}+s_{13} \sigma_{z},
\end{aligned}
$$

a)

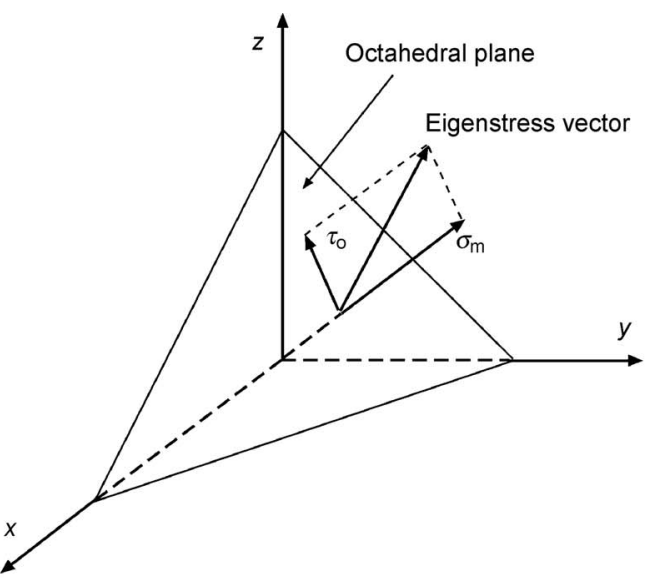

b)

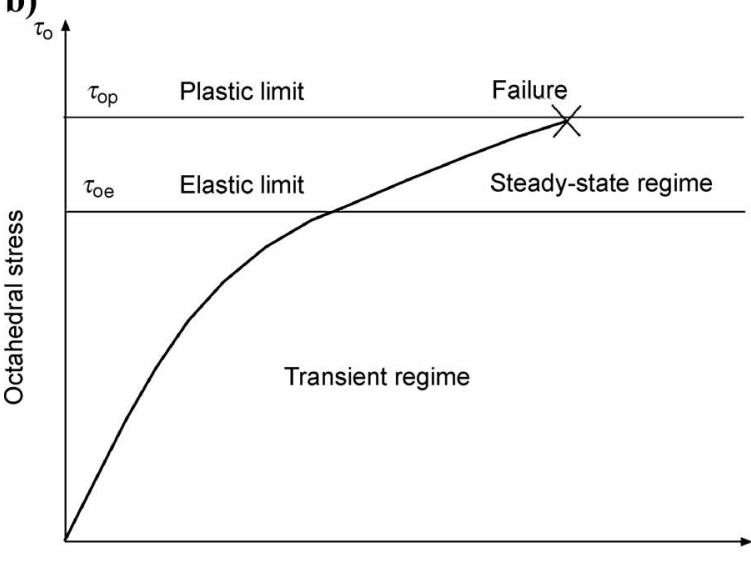

Effective strain

Figure 2. (a) Mean and octahedral stresses and (b) octahedral stress versus octahedral strain. The octahedral-stress vector, which is a measure of the shear deformation, lies on the octahedral plane. The normal to this surface makes the same angle with the direction of the three principal stresses $\sigma_{1}, \sigma_{2}$, and $\sigma_{3}$. The octahedral stress is proportional to $\sqrt{\left(\sigma_{1}-\sigma_{2}\right)^{2}+\left(\sigma_{1}-\sigma_{3}\right)^{2}+\left(\sigma_{2}-\sigma_{3}\right)^{2}}$ while the octaedral strain is proportional to $\sqrt{\left(\epsilon_{1}-\epsilon_{2}\right)^{2}+\left(\epsilon_{1}-\epsilon_{3}\right)^{2}+\left(\epsilon_{2}-\epsilon_{3}\right)^{2}}$, where $\epsilon_{1}, \epsilon_{2}$, and $\epsilon_{3}$ are the principal strains. 


$$
\epsilon_{z}=s_{13}\left(\sigma_{r}+\sigma_{\theta}\right)+s_{33} \sigma_{z}
$$

where

$$
\begin{aligned}
& s_{11}=\frac{1}{2}\left(\frac{c_{33}}{c^{2}}+\frac{1}{c_{11}-c_{12}}\right), \\
& s_{12}=\frac{1}{2}\left(\frac{c_{33}}{c^{2}}-\frac{1}{c_{11}-c_{12}}\right), \\
& s_{13}=-\frac{c_{13}}{c^{2}},
\end{aligned}
$$

and

$$
s_{33}=\frac{c_{11}+c_{12}}{c^{2}}
$$

are the compliance coefficients, $c_{i j}$ are the elastic stiffnesses, and

$$
c^{2}=c_{33}\left(c_{11}+c_{12}\right)-2 c_{13}^{2}
$$

(Auld, 1991).

In the isotropic limit, we have

$$
s_{11}=s_{33}=\frac{1}{Y}, \quad s_{12}=s_{13}=-\frac{\nu}{Y}
$$

where $Y$ and $\nu$ are the Young modulus and Poisson ratio, given by

$$
\begin{gathered}
Y=\frac{9 k \mu}{3 k+\mu}, \quad \text { and } \\
\nu=\frac{3 k-2 \mu}{2(3 k+\mu)}=\frac{1}{2}\left(1-\frac{1}{1 / 3+k / \mu}\right),
\end{gathered}
$$

where $k$ and $\mu$ are the bulk and shear moduli, respectively. Because $\sigma_{h}=\sigma_{H}, \sigma_{r \theta}=0$ and the equilibrium condition before drilling is

$$
\frac{\partial \sigma_{r}}{\partial r}=\frac{\sigma_{\theta}-\sigma_{r}}{r}
$$

and the strain components are

$$
\epsilon_{r}=\frac{\partial u_{r}}{\partial r} \text { and } \epsilon_{\theta}=\frac{u_{r}}{r}
$$

(Jaeger and Cook, 1969; Karasudhi, 1991), where $u_{r}$ is the radial displacement.

Assuming that the stress in the $z$-direction is not affected by the drilling, the third equation 7 yields

$$
\sigma_{z}=-\frac{s_{13}}{s_{33}}\left(\sigma_{r}+\sigma_{\theta}\right)+C,
$$

where $C=\epsilon_{z} / s_{33}$ is a constant. Substituting equation 14 into the first two equations of 7 and using 13 gives

$$
\frac{\partial u_{r}}{\partial r}=\left(s_{11}-a\right) \sigma_{r}+\left(s_{12}-a\right) \sigma_{\theta}+s_{13} C,
$$

and

$$
\frac{u_{r}}{r}=\left(s_{12}-a\right) \sigma_{r}+\left(s_{11}-a\right) \sigma_{\theta}+s_{13} C,
$$

where

$$
a=\frac{s_{13}^{2}}{s_{33}} .
$$

Differentiating equation 16 with respect to $r$, and using equation 15 yields

$$
\left(\sigma_{r}-\sigma_{\theta}\right)\left(s_{11}-s_{12}\right)=r\left[\left(s_{12}-a\right) \frac{\partial \sigma_{r}}{\partial r}+\left(s_{11}-a\right) \frac{\partial \sigma_{\theta}}{\partial r}\right] .
$$

Substituting this equation into equation 12 gives

$$
\frac{\partial \sigma_{r}}{\partial r}+\frac{\partial \sigma_{\theta}}{\partial r}=0
$$

Integration of equation 19 with respect to $r$ gives

$$
\sigma_{r}+\sigma_{\theta}=2 C_{1}
$$

where $C_{1}$ is an integration constant. From equation 12, we have

$$
\frac{\partial \sigma_{r}}{\partial r}=\frac{1}{r}\left(-2 \sigma_{r}+2 C_{1}\right)
$$

Integration of equation 21 results in

$$
\sigma_{r}=C_{1}+\frac{C_{2}}{r^{2}}
$$

where $C_{2}$ is another integration constant. Using equations 14 and 20 , we obtain the other two stress components,

$$
\sigma_{\theta}=C_{1}-\frac{C_{2}}{r^{2}}
$$

and

$$
\sigma_{z}=-\frac{2 s_{13} C_{1}}{s_{33}}+C
$$

These equations have the same functional form as those for isotropic rock salt (Infante and Chenevert, 1986; Cristescu, 1989), although this is not obvious from the beginning mathematical viewpoint. This equivalence results from the azimuthal symmetry in the horizontal plane. 
The boundary conditions are

$$
\begin{aligned}
& \sigma_{r}=-p \text { at } r=r_{0}, \\
& \sigma_{r}=\sigma_{\theta}=\sigma_{h} \text { at } r=\infty, \\
& \sigma_{z}=\sigma_{v} \text { at } r=\infty
\end{aligned}
$$

(see equation 3), where $r_{0}$ is the borehole radius and $p$ is the well pressure. Using equations 22-24, we obtain $C_{1}=\sigma_{h}, C_{2}=-(p$ $\left.+\sigma_{h}\right) r_{0}^{2}$, and

$$
\begin{aligned}
& \sigma_{r}=\sigma_{h}-\left(p+\sigma_{h}\right)\left(\frac{r_{0}}{r}\right)^{2}, \\
& \sigma_{\theta}=\sigma_{h}+\left(p+\sigma_{h}\right)\left(\frac{r_{0}}{r}\right)^{2},
\end{aligned}
$$

and

$$
\sigma_{z}=\sigma_{v}
$$

Therefore, the strain components in equation 7 are given by

$$
\begin{aligned}
& \epsilon_{r}=\frac{1}{c^{2}}\left(c_{33} \sigma_{h}-c_{13} \sigma_{v}\right)-\frac{1}{c_{11}-c_{12}}\left(p+\sigma_{h}\right)\left(\frac{r_{0}}{r}\right)^{2}, \\
& \epsilon_{\theta}=\frac{1}{c^{2}}\left(c_{33} \sigma_{h}-c_{13} \sigma_{v}\right)+\frac{1}{c_{11}-c_{12}}\left(p+\sigma_{h}\right)\left(\frac{r_{0}}{r}\right)^{2},
\end{aligned}
$$

and

$$
\epsilon_{z}=\frac{1}{c^{2}}\left[-2 c_{13} \sigma_{h}+\left(c_{11}+c_{12}\right) \sigma_{v}\right]
$$

where we have used equations 8 . The radial displacement is obtained from equation 13:

$$
u_{r}=\frac{1}{c^{2}}\left(c_{33} \sigma_{h}-c_{13} \sigma_{v}\right) r+\frac{1}{c_{11}-c_{12}}\left(p+\sigma_{h}\right)\left(\frac{r_{0}^{2}}{r}\right) \text {. }
$$

The first term corresponds to the the displacement before drilling when $\sigma_{r}=\sigma_{\theta}=\sigma_{h}$ and $\sigma_{z}=\sigma_{v}$. After drilling, the relative displacement is given by the second term.

The mean and octahedral stresses in equations 1 and 2, are given, respectively, by equation 5 and

$$
\tau_{o}=\frac{\sqrt{2}}{3} \sqrt{3\left(p+\sigma_{h}\right)^{2}\left(\frac{r_{0}}{r}\right)^{4}+\left(\sigma_{h}-\sigma_{v}\right)^{2}} .
$$

The maximum octahedral stress occurs at the borehole wall, and this value should be less than $\tau_{o e}$, the octahedral shear stress limit for elastic (transient) behavior. Hence, the borehole pressure to maintain the elastic behavior of the salt formation should satisfy

$$
\begin{aligned}
\sigma_{h}-\sqrt{\frac{3}{2} \tau_{o e}^{2}-\frac{1}{3}\left(\sigma_{h}-\sigma_{v}\right)^{2}} \\
\leq-p \leq \sigma_{h}+\sqrt{\frac{3}{2} \tau_{o e}^{2}-\frac{1}{3}\left(\sigma_{h}-\sigma_{v}\right)^{2}} .
\end{aligned}
$$

This range of wellbore pressures is the same as the isotropic model (Infante and Chenevert, 1986). The differences of the isotropic case are in the expressions of the radial displacement and strain components, which depend on the values of the elastic constants. The borehole will be stabilized perfectly for an isotropic (or even anisotropic) medium when the borehole pressure is equal to the isotropic tectonic stress. Obviously, this will not apply to an anisotropic state of stress if the medium is linearly viscoelastic at all octahedral stresses. It will be stabilized if the medium is elastic below the $\tau_{o e}$ octahedral stress. There will be a range of borehole pressures to keep the octahedral stress below $\tau_{o e}$ or $\tau_{o p}$ for an isotropic medium under anisotropic stress even though the range would be different than that for the anisotropic medium.

\section{Transient-creep effects in isotropic rock}

Viscoelastic flow (creep) is present at all levels of octahedral stress but becomes important when $\tau_{o}$ exceeds the elastic octahedral stress $\tau_{o e}$. Therefore, we assume that below this level we only have transient creep. The $\tau_{o}$ used to apply this criterion is obtained from equation 75 (see below), i.e., the maximum octahedral stress at the borehole wall after drilling. In order to include transient creep, we consider the generalized Zener viscoelastic strain-stress relation (Ben-Menahem and Singh, 1981; Klausner, 1991; Carcione, 2001). This model gives relaxation and creep functions in agreement with experiments (Zener, 1948). We define the deviatoric stress and strain tensors as

$$
\gamma_{i j}=\sigma_{i j}-\frac{1}{3} \sigma \delta_{i j}
$$

and

$$
d_{i j}=\epsilon_{i j}-\frac{1}{3} \vartheta \delta_{i j}
$$

respectively, where, according to the Einstein summation convention of repeated indices, $\sigma=\sigma_{i i}$ and $\vartheta=\epsilon_{i i}$ are the traces of the stress and strain tensors.

The one-mechanism 3D form for isotropic viscoelastic media is

$$
3 k_{0}\left(\vartheta+\tau_{\epsilon}^{(1)} \dot{\vartheta}\right)=\sigma+\tau_{\sigma}^{(1)} \dot{\sigma}
$$

and

$$
2 \mu_{0}\left(d_{i j}+\tau_{\epsilon}^{(2)} \dot{d}_{i j}\right)=\gamma_{i j}+\tau_{\sigma}^{(2)} \dot{\gamma}_{i j}
$$

(Ben-Menahem and Singh, 1981), where $k_{0}$ and $\mu_{0}$ are the relaxed bulk and shear moduli; $\tau_{\epsilon}^{(m)}$ and $\tau_{\sigma}^{(m)}$ are material relaxation times, corresponding to dilatational $(m=1)$ and shear $(m=2)$ deformations; and a dot above a variable denotes time differentiation. The unrelaxed moduli are 


$$
k_{\infty}=\left(\frac{\tau_{\epsilon}^{(1)}}{\tau_{\sigma}^{(1)}}\right) k_{0}, \quad \text { and } \mu_{\infty}=\left(\frac{\tau_{\epsilon}^{(2)}}{\tau_{\sigma}^{(2)}}\right) \mu_{0}
$$

with $\tau_{\sigma}^{(m)}<\tau_{\epsilon}^{(m)}$.

In the frequency domain, the time derivative is replaced by $i \omega$, where $\omega$ is the angular frequency, and equations 33 and 34 take the form

$$
\vartheta=\frac{1}{3 k_{0}}\left(\frac{1+i \omega \tau_{\sigma}^{(1)}}{1+i \omega \tau_{\epsilon}^{(1)}}\right) \sigma \equiv \frac{\sigma}{3 k}
$$

and

$$
d_{i j}=\frac{1}{2 \mu_{0}}\left(\frac{1+i \omega \tau_{\sigma}^{(2)}}{1+i \omega \tau_{\epsilon}^{(2)}}\right) \gamma_{i j} \equiv \frac{\gamma_{i j}}{2 \mu}
$$

where $k(\omega)$ and $\mu(\omega)$ are the bulk and shear complex moduli, respectively.

The relaxation times can be expressed as

$$
\tau_{\epsilon}^{(m)}=\frac{\tau_{0}}{Q_{0}^{(m)}}\left[\sqrt{Q_{0}^{(m) 2}+1}+1\right], \quad \tau_{\sigma}^{(m)}=\tau_{\epsilon}^{(m)}-\frac{2 \tau_{0}}{Q_{0}^{(m)}},
$$

where $\tau_{0}$ is a relaxation time such that $\omega_{0}=1 / \tau_{0}$ is the center frequency of the relaxation peak and $Q_{0}^{(m)}$ is the minimum quality factor, which can be obtained from the experimental relaxation times. The overall dilatational and shear quality factors are given by $Q_{d}$ $=\operatorname{Re}(k) / \operatorname{Im}(k)$ and $Q_{s}=\operatorname{Re}(\mu) / \operatorname{Im}(\mu)$, respectively (Ben-Menahem and Singh, 1981; Carcione, 2001), where Re and Im denote real and imaginary parts.

The stress components after drilling for an elastic medium have been obtained by Kirsch (1898) [see Jaeger and Cook, (1969); Cristescu, (1989); and Charlez, (1991)]:

$$
\begin{aligned}
\sigma_{r r}= & \frac{\left(\sigma_{H}+\sigma_{h}\right)}{2}\left(1-\frac{r_{0}^{2}}{r^{2}}\right)+\frac{\left(\sigma_{H}-\sigma_{h}\right)}{2} \\
& \times\left(1-\frac{4 r_{0}^{2}}{r^{2}}+\frac{3 r_{0}^{4}}{r^{4}}\right) \cos 2 \theta-\frac{p r_{0}^{2}}{r^{2}}, \\
\sigma_{\theta \theta}= & \frac{\left(\sigma_{H}+\sigma_{h}\right)}{2}\left(1+\frac{r_{0}^{2}}{r^{2}}\right)-\frac{\left(\sigma_{H}-\sigma_{h}\right)}{2} \\
& \times\left(1+\frac{3 r_{0}^{4}}{r^{4}}\right) \cos 2 \theta+\frac{p r_{0}^{2}}{r^{2}}, \\
\sigma_{r \theta}= & -\frac{\left(\sigma_{H}-\sigma_{h}\right)}{2}\left(1+\frac{2 r_{0}^{2}}{r^{2}}-\frac{3 r_{0}^{4}}{r^{4}}\right) \sin 2 \theta,
\end{aligned}
$$

and

$$
\sigma_{z z}=\sigma_{v}-\nu\left(\sigma_{H}-\sigma_{h}\right) \frac{2 r_{0}^{2}}{r^{2}} \cos 2 \theta
$$

At the borehole wall $\left(r=r_{0}\right)$ and after drilling,

$$
\begin{aligned}
\sigma_{r r} & =-p, \\
\sigma_{\theta \theta} & =\left(\sigma_{H}+\sigma_{h}\right)-2\left(\sigma_{H}-\sigma_{h}\right) \cos 2 \theta+p, \\
\sigma_{r \theta} & =0,
\end{aligned}
$$

and

$$
\sigma_{z z}=\sigma_{v}-2 \nu\left(\sigma_{H}-\sigma_{h}\right) \cos 2 \theta
$$

Note that $\sigma_{\theta \theta}(\theta=0)=3 \sigma_{h}-\sigma_{H}+p<\sigma_{\theta \theta}(\theta=\pi / 2)=3 \sigma_{H}$ $-\sigma_{h}+p$, defining the minimum and maximum-stress concentrations at $\theta=0$ and $\theta=\pi / 2$, respectively.

The relative stresses are obtained by subtracting equations 3 from equations 39 :

$$
\begin{aligned}
\sigma_{r r}= & -\frac{\left(\sigma_{H}+\sigma_{h}\right)}{2} \frac{r_{0}^{2}}{r^{2}}-\frac{\left(\sigma_{H}-\sigma_{h}\right)}{2}\left(\frac{4 r_{0}^{2}}{r^{2}}-\frac{3 r_{0}^{4}}{r^{4}}\right) \cos 2 \theta \\
& -\frac{p r_{0}^{2}}{r^{2}}, \\
\sigma_{\theta \theta}= & \frac{\left(\sigma_{H}+\sigma_{h}\right)}{2} \frac{r_{0}^{2}}{r^{2}}-\frac{\left(\sigma_{H}-\sigma_{h}\right)}{2}\left(\frac{3 r_{0}^{4}}{r^{4}}\right) \cos 2 \theta+\frac{p r_{0}^{2}}{r^{2}}, \\
\sigma_{r \theta}= & -\frac{\left(\sigma_{H}-\sigma_{h}\right)}{2}\left(\frac{2 r_{0}^{2}}{r^{2}}-\frac{3 r_{0}^{4}}{r^{4}}\right) \sin 2 \theta,
\end{aligned}
$$

and

$$
\sigma_{z z}=-\nu\left(\sigma_{H}-\sigma_{h}\right)\left(\frac{2 r_{0}^{2}}{r^{2}}\right) \cos 2 \theta
$$

Invoking the correspondence principle (Ben-Menahem and Singh, 1981; Carcione, 2001), the viscoelastic frequency-domain, strainstress relations have the same form of the elastic, time-domain, strain-stress relations. Assuming isotropy, the frequency-domain strain-stress relations are

$$
\begin{aligned}
& \epsilon_{r r}=\frac{1}{Y}\left[\sigma_{r r}-\nu\left(\sigma_{\theta \theta}+\sigma_{z z}\right)\right], \\
& \epsilon_{\theta \theta}=\frac{1}{Y}\left[\sigma_{\theta \theta}-\nu\left(\sigma_{r r}+\sigma_{z z}\right)\right], \\
& \epsilon_{z z}=\frac{1}{Y}\left[\sigma_{z z}-\nu\left(\sigma_{r r}+\sigma_{\theta \theta}\right)\right], \\
& \epsilon_{r \theta}=\frac{1}{2 \mu} \sigma_{r \theta}
\end{aligned}
$$

(Ben-Menahem and Singh, 1981), where here $Y$ and $\nu$ are complex and frequency dependent, through equations 11, 36, and 37. Because for plane strain, $\epsilon_{z z}=0$, equations 42 become

$$
\begin{aligned}
\epsilon_{r r} & =\frac{1}{2 \mu} \sigma_{r r}-\frac{\nu}{2 \mu}\left(\sigma_{r r}+\sigma_{\theta \theta}\right) \\
& =\frac{1}{4 \mu}\left(\sigma_{r r}-\sigma_{\theta \theta}\right)+\frac{3}{4}\left(\frac{1}{\mu+3 k}\right)\left(\sigma_{r r}+\sigma_{\theta \theta}\right),
\end{aligned}
$$




$$
\begin{aligned}
\epsilon_{\theta \theta} & =\frac{1}{2 \mu} \sigma_{\theta \theta}-\frac{\nu}{2 \mu}\left(\sigma_{r r}+\sigma_{\theta \theta}\right) \\
& =\frac{1}{4 \mu}\left(\sigma_{\theta \theta}-\sigma_{r r}\right)+\frac{3}{4}\left(\frac{1}{\mu+3 k}\right)\left(\sigma_{r r}+\sigma_{\theta \theta}\right),
\end{aligned}
$$

and

$$
\epsilon_{r \theta}=\frac{1}{2 \mu} \sigma_{r \theta},
$$

where we have used equation 11. From equations 36 and 37 and using partial fractions, we have

$$
\begin{aligned}
\frac{1}{\mu} & =\frac{1}{\mu_{0}}\left(\frac{1+i \omega \tau_{\sigma}^{(2)}}{1+i \omega \tau_{\epsilon}^{(2)}}\right), \\
\frac{1}{\mu+3 k} & =\left[\mu_{0}\left(\frac{1+i \omega \tau_{\epsilon}^{(2)}}{1+i \omega \tau_{\sigma}^{(2)}}\right)+3 k_{0}\left(\frac{1+i \omega \tau_{\epsilon}^{(1)}}{1+i \omega \tau_{\sigma}^{(1)}}\right)\right]^{-1} \\
& =\frac{1}{\mu_{\infty}+3 k_{\infty}}+\frac{\omega_{1}}{b_{1}\left(\omega_{1}+i \omega\right)}+\frac{\omega_{2}}{b_{2}\left(\omega_{2}+i \omega\right)},
\end{aligned}
$$

where

$$
\begin{aligned}
b_{1} & =\frac{\omega_{1}\left(\omega_{1}-\omega_{2}\right)}{a+b \omega_{1}-c \omega_{1}^{2}}, \quad b_{2}=\frac{\omega_{2}\left(\omega_{2}-\omega_{1}\right)}{a+b \omega_{2}-c \omega_{2}^{2}}, \\
a & =-\frac{1}{3 k_{0} \tau_{\epsilon}^{(1)} \tau_{\sigma}^{(2)}+\mu_{0} \tau_{\epsilon}^{(2)} \tau_{\sigma}^{(1)}}, \\
b & =-a\left(\tau_{\sigma}^{(1)}+\tau_{\sigma}^{(2)}\right), \quad c=-a \tau_{\sigma}^{(1)} \tau_{\sigma}^{(2)}, \\
\omega_{1} & =-\frac{a}{2}\left[d-\sqrt{d^{2}+\frac{4\left(3 k_{0}+\mu_{0}\right)}{a}}\right], \\
\omega_{2} & =-\frac{a}{2}\left[d+\sqrt{d^{2}+\frac{4\left(3 k_{0}+\mu_{0}\right)}{a}}\right]
\end{aligned}
$$

and

$$
d=3 k_{0}\left(\tau_{\epsilon}^{(1)}+\tau_{\sigma}^{(2)}\right)+\mu_{0}\left(\tau_{\epsilon}^{(2)}+\tau_{\sigma}^{(1)}\right)
$$

The quantity inside the square root in equation 46 can be shown to be always positive under the conditions $k_{0}>0, \mu_{0}>0, \tau_{\epsilon}^{(m)}$ $>\tau_{\sigma}^{(m)}, \tau_{\epsilon}^{(m)}>0$, and $\tau_{\sigma}^{(m)}>0$.

The shear modulus is related to the creep function $\chi_{2}$ by

$$
\frac{1}{\mu}=\mathcal{F}\left(\dot{\chi}_{2}\right)
$$

(Carcione, 2001), where $\mathcal{F}$ takes Fourier transform from the time domain to the frequency domain, and

$$
\chi_{2}=\frac{1}{\mu_{0}}\left[1-\left(1-\frac{\tau_{\sigma}^{(2)}}{\tau_{\epsilon}^{(2)}}\right) \exp \left(-t / \tau_{\epsilon}^{(2)}\right)\right] H(t),
$$

where $H(t)$ is the Heaviside function. A Fourier transform of equation 45 to the time domain gives the response function,

$$
\begin{aligned}
\phi(t)= & \frac{\delta(t)}{\mu_{\infty}+3 k_{\infty}}+\left[\left(\frac{\omega_{1}}{b_{1}}\right) \exp \left(-\omega_{1} t\right)\right. \\
& \left.+\left(\frac{\omega_{2}}{b_{2}}\right) \exp \left(-\omega_{2} t\right)\right] H(t),
\end{aligned}
$$

where $\delta(t)$ is Dirac's function. The corresponding creep function is

$$
\begin{aligned}
\chi(t)= & \left\{\frac{1}{\mu_{\infty}+3 k_{\infty}}+\frac{1}{b_{1}}\left[1-\exp \left(-\omega_{1} t\right)\right]\right. \\
& \left.+\frac{1}{b_{2}}\left[1-\exp \left(-\omega_{2} t\right)\right]\right\} H(t),
\end{aligned}
$$

such that $\dot{\chi}=\phi$, and the following relation holds

$$
\frac{1}{\mu+3 k}=\mathcal{F}(\dot{\chi})
$$

Hence, the time-domain strain-stress relations in equation 43 are

$$
\begin{aligned}
\epsilon_{r r} & =\frac{1}{4} \chi_{2} *\left(\dot{\sigma}_{r r}-\dot{\sigma}_{\theta \theta}\right)+\frac{3}{4} \chi *\left(\dot{\sigma}_{r r}+\dot{\sigma}_{\theta \theta}\right), \\
\epsilon_{\theta \theta} & =\frac{1}{4} \chi_{2} *\left(\dot{\sigma}_{\theta \theta}-\dot{\sigma}_{r r}\right)+\frac{3}{4} \chi *\left(\dot{\sigma}_{r r}+\dot{\sigma}_{\theta \theta}\right),
\end{aligned}
$$

and

$$
\epsilon_{r \theta}=\frac{1}{2} \chi_{2} * \dot{\sigma}_{r \theta}
$$

where the $*$ denotes time convolution. In a creep process, the stresses remain constant and have the form $\sigma_{0} H(t)$, where $\sigma_{0}$ is constant. It follows that equations 52 can be written as

$$
\begin{aligned}
\epsilon_{r r} & =\frac{1}{4} \chi_{2}\left(\sigma_{r r}-\sigma_{\theta \theta}\right)+\frac{3}{4} \chi\left(\sigma_{r r}+\sigma_{\theta \theta}\right), \\
\epsilon_{\theta \theta} & =\frac{1}{4} \chi_{2}\left(\sigma_{\theta \theta}-\sigma_{r r}\right)+\frac{3}{4} \chi\left(\sigma_{r r}+\sigma_{\theta \theta}\right),
\end{aligned}
$$

and

$$
\epsilon_{r \theta}=\frac{1}{2} \chi_{2} \sigma_{r \theta}
$$

where $\sigma_{r r}, \sigma_{\theta \theta}$, and $\sigma_{r \theta}$ are given by equation 39 to obtain the total strain components, and by equation 41 to obtain the relative-strain components. 
The strain-displacement relations in cylindrical coordinates are

$$
\begin{aligned}
\epsilon_{r r} & =\frac{\partial u_{r}}{\partial r}, \\
\epsilon_{\theta \theta} & =\frac{1}{r}\left(u_{r}+\frac{\partial u_{\theta}}{\partial \theta}\right),
\end{aligned}
$$

and

$$
\epsilon_{r \theta}=\frac{1}{2}\left[\frac{1}{r}\left(\frac{\partial u_{r}}{\partial \theta}-u_{\theta}\right)+\frac{\partial u_{\theta}}{\partial r}\right]
$$

Using the relative stress components in equation 41 and integrating the first equation 54 yields

$$
\begin{aligned}
u_{r}= & \frac{1}{4} \chi_{2}\left\{\left(\sigma_{H}+\sigma_{h}\right)\left(\frac{r_{0}^{2}}{r}\right)+\left(\sigma_{H}-\sigma_{h}\right)\left(\frac{2 r_{0}^{2}}{r}-\frac{r_{0}^{4}}{r^{3}}\right) \cos 2 \theta\right. \\
& \left.+\frac{2 p r_{0}^{2}}{r}\right\}+\frac{3}{2} \chi\left[\left(\sigma_{H}-\sigma_{h}\right)\left(\frac{r_{0}^{2}}{r}\right) \cos 2 \theta\right]+\frac{d f(\theta)}{d \theta},
\end{aligned}
$$

where $f(\theta)$ depends only on $\theta$. The second equation 54 implies

$$
u_{\theta}=\int\left(r \epsilon_{\theta \theta}-u_{r}\right) d \theta
$$

Using equations 53 and 55, we obtain

$$
\begin{aligned}
u_{\theta}= & -\frac{1}{4}\left(\sigma_{H}-\sigma_{h}\right)\left(\frac{r_{0}^{2}}{r}\right)\left[\chi_{2}\left(\frac{r_{0}^{2}}{r^{2}}\right)+6 \chi\right] \sin 2 \theta-f(\theta) \\
& +g(r),
\end{aligned}
$$

where $g(r)$ is independent of $\theta$. It can be verified that $\epsilon_{r \theta}$ calculated from equations 54 and 53 (see equation 41) are equivalent if

$$
\frac{d^{2} f(\theta)}{d \theta^{2}}+f-g+\frac{d g(r)}{d r}=0
$$

The solution is

$$
\begin{aligned}
f(\theta)= & \alpha_{1} \sin \theta+\alpha_{2} \cos \theta-\alpha_{0}, \\
& \text { and } g(r)=\alpha_{3} \exp (r)-\alpha_{0},
\end{aligned}
$$

where the constants equal $\alpha$. These constants must be zero, because the relative displacements should vanish at $r \rightarrow \infty$. Thus, the relative displacements are

$$
\begin{aligned}
u_{r}(r, \theta, t)= & \frac{1}{4} \chi_{2}(t)\left\{\left(\sigma_{H}+\sigma_{h}\right)\left(\frac{r_{0}^{2}}{r}\right)\right. \\
& \left.+\left(\sigma_{H}-\sigma_{h}\right)\left(\frac{2 r_{0}^{2}}{r}-\frac{r_{0}^{4}}{r^{3}}\right) \cos 2 \theta+\frac{2 p r_{0}^{2}}{r}\right\}
\end{aligned}
$$

$$
+\frac{3}{2} \chi(t)\left[\left(\sigma_{H}-\sigma_{h}\right)\left(\frac{r_{0}^{2}}{r}\right) \cos 2 \theta\right]
$$

and

$$
u_{\theta}(r, \theta, t)=-\frac{1}{4}\left(\sigma_{H}-\sigma_{h}\right)\left(\frac{r_{0}^{2}}{r}\right)\left[\chi_{2}(t)\left(\frac{r_{0}^{2}}{r^{2}}\right)+6 \chi(t)\right] \sin 2 \theta,
$$

where $\chi_{2}$ and $\chi$ are given by equations 48 and 50, respectively.

If $t \rightarrow \infty, \chi_{2} \rightarrow 1 / \mu_{0}, \chi \rightarrow 1 /\left(\mu_{0}+3 k_{0}\right)$, and the relative displacements at the borehole wall satisfy

$$
\begin{aligned}
\frac{u_{r}\left(r_{0}, \theta\right)}{r_{0}}= & \frac{1}{4 \mu_{0}}\left(\sigma_{H}+\sigma_{h}+2 p\right) \\
& +\frac{7 \mu_{0}+3 k_{0}}{4 \mu_{0}\left(\mu_{0}+3 k_{0}\right)}\left(\sigma_{H}-\sigma_{h}\right) \cos 2 \theta
\end{aligned}
$$

and

$$
\frac{u_{\theta}\left(r_{0}, \theta\right)}{r_{0}}=-\frac{7 \mu_{0}+3 k_{0}}{4 \mu_{0}\left(\mu_{0}+3 k_{0}\right)}\left(\sigma_{H}-\sigma_{h}\right) \sin 2 \theta .
$$

\section{Optimal mud weight and borehole shrinkage}

Let us calculate the optimal mud weights or optimal borehole pressures to avoid borehole closure. The maximum-stress concentration at the borehole wall occurs at $\theta=\pi / 2$, where equations 40 become

$$
\begin{aligned}
\sigma_{r r} & =-p, \\
\sigma_{\theta \theta} & =3 \sigma_{H}-\sigma_{h}+p, \\
\sigma_{r \theta} & =0,
\end{aligned}
$$

and

$$
\sigma_{z z}=\sigma_{v}+2 \nu\left(\sigma_{H}-\sigma_{h}\right)
$$

We consider the unrelaxed and the relaxed states. In the latter case, we should replace $\nu$ by $\nu_{0}=\left[1-1 /\left(1 / 3-k_{0} / \mu_{0}\right)\right] / 2$, i.e., the relaxed Poisson ratio. The maximum octahedral stress after drilling is obtained by substituting the preceding equations into equation 2 . This gives the following relation:

$$
\begin{aligned}
& -9 \tau_{o}^{2}+6 p^{2}+6\left(3 \sigma_{H}-\sigma_{h}\right) p+2\left(3 \sigma_{H}-\sigma_{h}-\sigma_{z z}\right)^{2} \\
& +2\left(3 \sigma_{H}-\sigma_{h}\right) \sigma_{z z}=0
\end{aligned}
$$

where $\sigma_{z z}$ is given by equation 62 . The maximum octahedral stress should be less than $\tau_{o e}$, the octahedral-stress limit for unrelaxed (elastic) behavior or less than $\tau_{o r}$, the octahedral-stress limit for relaxed behavior if $\nu$ is replaced by its relaxed value $\nu_{0}$. Solving equation 63 for $p$, we obtain the following condition for the borehole pressure: 


$$
p_{1} \leq-p \leq p_{2}
$$

where

$$
\begin{aligned}
p_{1}= & \frac{3 \sigma_{H}-\sigma_{h}}{2} \\
& -\frac{1}{6} \sqrt{54 \tau_{o}^{2}-3\left(3 \sigma_{H}-\sigma_{h}\right)^{2}-12 \sigma_{z z}^{2}+12\left(3 \sigma_{H}-\sigma_{h}\right) \sigma_{z z}},
\end{aligned}
$$

and

$$
\begin{aligned}
p_{2}= & \frac{3 \sigma_{H}-\sigma_{h}}{2} \\
& +\frac{1}{6} \sqrt{54 \tau_{o}^{2}-3\left(3 \sigma_{H}-\sigma_{h}\right)^{2}-12 \sigma_{z z}^{2}+12\left(3 \sigma_{H}-\sigma_{h}\right) \sigma_{z z}} .
\end{aligned}
$$

If $\sigma_{H}=\sigma_{h}$, we obtain condition equation 30 .

In the following, we determine the change in the shape of the borehole cross section (Cristescu, 1989). A point in this surface, with initial coordinates $x_{0}=r_{0} \cos \theta$ and $y_{0}=r_{0} \sin \theta$, has the following coordinates at time $t$ :

$$
x(t)=x_{0}-\left[u_{r}\left(r_{0}, \theta, t\right) \cos \theta-u_{\theta}\left(r_{0}, \theta, t\right) \sin \theta\right]
$$

and

$$
y(t)=y_{0}-\left[u_{r}\left(r_{0}, \theta, t\right) \sin \theta+u_{\theta}\left(r_{0}, \theta, t\right) \cos \theta\right] .
$$

We have from equations 58 and 59:

$$
\begin{aligned}
u_{r}\left(r_{0}, \theta, t\right)= & \frac{1}{4} r_{0}\left(\sigma_{H}+\sigma_{h}+2 p\right) \chi_{2} \\
& +\frac{1}{4} r_{0}\left(\sigma_{H}-\sigma_{h}\right)\left(\chi_{2}+6 \chi\right) \cos 2 \theta
\end{aligned}
$$

and

$$
u_{\theta}\left(r_{0}, \theta, t\right)=-\frac{1}{4} r_{0}\left(\sigma_{H}-\sigma_{h}\right)\left(\chi_{2}+6 \chi\right) \sin 2 \theta .
$$

Substituting these equations into 67 gives

$$
\begin{aligned}
x= & r_{0} \cos \theta\left[1-\frac{1}{4}\left(\sigma_{H}+\sigma_{h}+2 p\right) \chi_{2}\right. \\
& \left.-\frac{1}{4}\left(\sigma_{H}-\sigma_{h}\right)\left(\chi_{2}+6 \chi\right)\right], \\
y= & r_{0} \sin \theta\left[1-\frac{1}{4}\left(\sigma_{H}+\sigma_{h}+2 p\right) \chi_{2}\right. \\
& \left.+\frac{1}{4}\left(\sigma_{H}-\sigma_{h}\right)\left(\chi_{2}+6 \chi\right)\right],
\end{aligned}
$$

which are the parametric equations of an ellipse. The minor and major semiaxes vary with time and are given by

$$
\begin{aligned}
a(t)= & r_{0}\left[1-\frac{1}{4}\left(\sigma_{H}+\sigma_{h}+2 p\right) \chi_{2}(t)\right. \\
& \left.-\frac{1}{4}\left(\sigma_{H}-\sigma_{h}\right)\left(\chi_{2}+6 \chi(t)\right)\right]
\end{aligned}
$$

and

$$
\begin{aligned}
b(t)= & r_{0}\left[1-\frac{1}{4}\left(\sigma_{H}+\sigma_{h}+2 p\right) \chi_{2}(t)\right. \\
& \left.+\frac{1}{4}\left(\sigma_{H}-\sigma_{h}\right)\left(\chi_{2}+6 \chi(t)\right)\right] .
\end{aligned}
$$

The difference between the major and minor semiaxis is

$$
\Delta a(t)=b(t)-a(t)=\frac{r_{0}}{2}\left(\sigma_{H}-\sigma_{h}\right)\left[\chi_{2}(t)+6 \chi(t)\right] .
$$

At $t=0$ and $t=\infty$, the minor semiaxis is

$$
\begin{aligned}
a(0)= & r_{0}\left[1-\frac{1}{4 \mu_{\infty}}\left(\sigma_{H}+\sigma_{h}+2 p\right)\right. \\
& \left.-\frac{7 \mu_{\infty}+3 k_{\infty}}{4 \mu_{\infty}\left(\mu_{\infty}+3 k_{\infty}\right)}\left(\sigma_{H}-\sigma_{h}\right)\right], \\
a(\infty)= & r_{0}\left[1-\frac{1}{4 \mu_{0}}\left(\sigma_{H}+\sigma_{h}+2 p\right)\right. \\
& \left.-\frac{7 \mu_{0}+3 k_{0}}{4 \mu_{0}\left(\mu_{0}+3 k_{0}\right)}\left(\sigma_{H}-\sigma_{h}\right)\right] .
\end{aligned}
$$

The major semiaxis is

$$
\begin{aligned}
b(0)= & r_{0}\left[1-\frac{1}{4 \mu_{\infty}}\left(\sigma_{H}+\sigma_{h}+2 p\right)\right. \\
& \left.+\frac{7 \mu_{\infty}+3 k_{\infty}}{4 \mu_{\infty}\left(\mu_{\infty}+3 k_{\infty}\right)}\left(\sigma_{H}-\sigma_{h}\right)\right]
\end{aligned}
$$

and

$$
\begin{aligned}
b(\infty)= & r_{0}\left[1-\frac{1}{4 \mu_{0}}\left(\sigma_{H}+\sigma_{h}+2 p\right)\right. \\
& \left.+\frac{7 \mu_{0}+3 k_{0}}{4 \mu_{0}\left(\mu_{0}+3 k_{0}\right)}\left(\sigma_{H}-\sigma_{h}\right)\right] .
\end{aligned}
$$

The viscoelastic moduli can be obtained from creep experiments. The more realistic test is the triaxial one, which brings the rock very close to the stress state existing around boreholes. A cylindrical sample is first subjected to hydrostatic pressure, and then the 
axial stress is increased and held constant for a very long time. During this interval, the variations of the axial and azimuthal strains are recorded. The relaxed and unrelaxed moduli can be obtained from the strain curves. In some cases, creep can be studied in situ (e.g., Ladanyi and Gill, 1983).

\section{Steady-state creep effects in isotropic rock}

Steady-state creep or significant viscoelastic flow can be described by using the Maxwell viscoelastic model (Ben-Menahem and Singh, 1981; Klausner, 1991; Carcione, 2001). We describe the deformation process by using the transient model of the previous section when $\tau_{o}<\tau_{o e}$, and the Maxwell model when $\tau_{o}>\tau_{o e}$, where $\tau_{o}$ is the maximum octahedral stress obtained from equation 63 , and assuming that the borehole pressure is equal to the vertical stress $-\sigma_{v}$. We obtain

$$
\tau_{o}=\frac{\sqrt{2}}{3} \sqrt{\left(3 \sigma_{H}-\sigma_{h}-2 \sigma_{v}\right)^{2}+4 \nu^{2}\left(\sigma_{H}-\sigma_{h}\right)^{2}-2 \nu\left(3 \sigma_{H}-\sigma_{h}-2 \sigma_{v}\right)\left(\sigma_{H}-\sigma_{h}\right)} .
$$

If $\sigma_{H}=\sigma_{h}$, we have $\tau_{o}=\sqrt{8}\left|\sigma_{h}-\sigma_{v}\right| / 3$.

We consider again the plane-strain equation 43:

$$
\begin{aligned}
& \epsilon_{r r}=\frac{1}{4 \mu}\left(\sigma_{r r}-\sigma_{\theta \theta}\right)+\frac{3}{4}\left(\frac{1}{\mu+3 k}\right)\left(\sigma_{r r}+\sigma_{\theta \theta}\right), \\
& \epsilon_{\theta \theta}=\frac{1}{4 \mu}\left(\sigma_{\theta \theta}-\sigma_{r r}\right)+\frac{3}{4}\left(\frac{1}{\mu+3 k}\right)\left(\sigma_{r r}+\sigma_{\theta \theta}\right),
\end{aligned}
$$

and

$$
\epsilon_{r \theta}=\frac{1}{2 \mu} \sigma_{r \theta}
$$

The corresponding complex compliance moduli are

$$
\frac{1}{\mu}=\frac{1}{\mu_{\infty}}+\frac{1}{i \omega \eta_{\mu}}
$$

and

$$
\frac{1}{k}=\frac{1}{k_{\infty}}+\frac{1}{i \omega \eta_{k}}
$$

where $\mu_{\infty}$ and $k_{\infty}$ are the unrelaxed moduli and $\eta_{\mu}$ and $\eta_{k}$ are viscosity parameters related to the shear and dilatational steady-state creep rates. Note that the Maxwell model is a particular case of the Zener model if the term $i \omega \tau_{\epsilon}^{(m)} \gg 1$ in equations 36 and 37, i.e., if the relaxation times are very large compared to the overall deformation process.

The shear modulus is related to the creep function $\chi_{2}$ by equation 47 , where

$$
\chi_{2}(t)=\frac{1}{\mu_{\infty}}\left(1+\frac{t}{\tau}\right) H(t), \quad \tau=\frac{\eta_{\mu}}{\mu_{\infty}} .
$$

(Ben-Menahem and Singh, 1981; Carcione, 2001) Using partial fractions, we have

$$
\frac{1}{\mu+3 k}=\frac{1}{\mu_{\infty}+3 k_{\infty}}+\frac{1}{i \omega \eta}+\frac{\omega_{1}}{b_{1}\left(\omega_{1}+i \omega\right)},
$$

where

$$
\eta=3 \eta_{k}+\eta_{\mu}, \quad \omega_{1}=\left(\frac{1}{k_{\infty}}+\frac{3}{\mu_{\infty}}\right)^{-1}\left(\frac{1}{\eta_{k}}+\frac{3}{\eta_{\mu}}\right),
$$

and

$$
b_{1}=\left[\left(\frac{\eta_{\mu}^{2}}{\mu_{\infty}}+\frac{3 \eta_{k}^{2}}{k_{\infty}}\right) \frac{1}{\eta^{2}}-\frac{1}{\mu_{\infty}+3 k_{\infty}}\right]^{-1} .
$$

A Fourier transform of equation 80 , to the time domain gives the following response function:

$$
\phi(t)=\frac{\delta(t)}{\mu_{\infty}+3 k_{\infty}}+\left[\frac{1}{\eta}+\left(\frac{\omega_{1}}{b_{1}}\right) \exp \left(-\omega_{1} t\right)\right] H(t) .
$$

The corresponding creep function is

$$
\chi(t)=\left\{\frac{1}{\mu_{\infty}+3 k_{\infty}}+\frac{t}{\eta}+\frac{1}{b_{1}}\left[1-\exp \left(-\omega_{1} t\right)\right]\right\} H(t) .
$$

The second term describes the steady-state creep process. The material responds instantaneously with the modulus $\mu_{\infty}+3 k_{\infty}$ and has a transient creep behavior with relaxation time equal to $1 / \omega_{1}$.

The strain-stress relations have the form equation 53, and the strain-displacement relations are given by equation 54 . Using the relative-stress components in equation 41 , we obtain the displacements in equations 58 and 59, where $\chi_{2}$ and $\chi$ are given by equations 79 and 84 , respectively.

\section{Optimal mud weight and borehole shrinkage}

According to equations $62-66$, at the onset of the steady-state creep process the mud weight must satisfy equation 64 , with $\nu$ $=\nu_{\infty}$. In order to obtain the mud weight, we need to calculate the stress component $\sigma_{z z}$ as a function of time by using equation 62. A partial-fraction decomposition of the frequency-dependent Poisson ratio (see equation 11) yields

$$
\nu=\nu_{\infty}+\frac{\omega_{1}}{b_{\nu}\left(\omega_{1}+i \omega\right)},
$$

where $\omega_{1}$ is given by equation 81 ,

$$
\nu_{\infty}=\frac{3 k_{\infty}-2 \mu_{\infty}}{2\left(3 k_{\infty}+\mu_{\infty}\right)},
$$

and 


$$
b_{\nu}=\frac{2 \eta}{3 \eta_{k}-2 \eta_{\mu}-2 \nu_{\infty} \eta}
$$

Then, we have

$$
\sigma_{z z}(t)=\sigma_{v}+2 \chi_{\nu}(t)\left(\sigma_{H}-\sigma_{h}\right)
$$

where

$$
\chi_{\nu}(t)=\left\{\nu_{\infty}+\frac{1}{b_{\nu}}\left[1-\exp \left(-\omega_{1} t\right)\right]\right\} H(t) .
$$

Therefore, the optimal mud weight should satisfy equation 64 with $\sigma_{z z}$ given by equation 88 . The relaxed value is obtained at $\omega=0$ or $t=\infty$, and it is

$$
\nu(0)=\chi_{\nu}(\infty)=\nu_{\infty}+1 / b_{\nu}=\frac{3 \eta_{k}-2 \eta_{\mu}}{2\left(3 \eta_{k}+\eta_{\mu}\right)} .
$$

Using equations 81 and 87 , we obtain

$$
\sigma_{z z}(t=\infty)=\sigma_{v}+\left(\frac{3 \eta_{k}-2 \eta_{\mu}}{3 \eta_{k}+\eta_{\mu}}\right)\left(\sigma_{H}-\sigma_{h}\right) .
$$

The major and minor semiaxes vary with time as in equation 71 , with $\chi_{2}$ and $\chi$ given by equations 79 and 84, respectively. At $t=0$, the minor semiaxis, $a(0)$, is given by the first equation 71 . The wall-closure time $t_{c}$ is obtained for $a\left(t_{c}\right)=0$, i.e., for

$$
\frac{1}{2}\left(\sigma_{H}+p\right) \chi_{2}\left(t_{c}\right)+\frac{3}{2}\left(\sigma_{H}-\sigma_{h}\right) \chi\left(t_{c}\right)=1,
$$

where

$$
\chi_{2}\left(t_{c}\right)=\frac{1}{\mu_{\infty}}+\frac{t_{c}}{\eta_{\mu}},
$$

and

$$
\chi\left(t_{c}\right)=\frac{1}{\mu_{\infty}+3 k_{\infty}}+\frac{t_{c}}{\eta}+\frac{1}{b_{1}}\left[1-\exp \left(-\omega_{1} t_{c}\right)\right] .
$$

If $\sigma_{H}=\sigma_{h}$,

$$
a(t)=b(t)=r_{0}\left[1-\frac{1}{2}\left(\sigma_{h}+p\right)\left(\frac{1}{\mu_{\infty}}+\frac{t_{c}}{\eta_{\mu}}\right)\right],
$$

and the condition of equation 92 can be solved analytically, giving

$$
t_{c}=\left(\frac{2}{\sigma_{h}+p}-\frac{1}{\mu_{\infty}}\right) \eta_{\mu} .
$$

If $p=-\sigma_{h}$, then $a(t)=r_{0}$ at all times. Wall closure occurs for $p$ $<-\sigma_{h}$.
Viscosities and steady-state creep rates

The viscosities $\eta_{k}$ and $\eta_{\mu}$ are related to the steady-state creep rates by

$$
\eta_{k}=\frac{\tau_{o}}{2 \dot{e}_{k}} \text { and } \eta_{\mu}=\frac{\tau_{o}}{2 \dot{e}_{\mu}}
$$

where $\tau_{o}$ is the octahedral stress in equation 75 , and $\dot{e}_{k}$ and $\dot{e}_{\mu}$ are the steady-state creep rates for dilatation and shear. These can be expressed as

$$
\dot{e}_{k}=A_{k} \tau_{o}^{n_{k}} \exp \left(-E_{k} / R T\right)
$$

and

$$
\dot{e}_{\mu}=A_{\mu} \tau_{o}^{n}{ }^{\mu} \exp \left(-E_{\mu} / R T\right)
$$

e.g., Gangi, (1983), where $A_{k}, A_{\mu}, n_{k}$, and $n_{\mu}$ are constants; $E_{k}$ and $E_{\mu}$ are the activation energies for dilatation and shear, respectively; $R$ is the gas constant; $T$ is the absolute temperature, and $\tau_{o}$ is given in MPa. The form of the empirical relations in equations 98 and 99 are determined by performing experiments at different strain rates, temperatures and/or stresses (e.g., Gangi, 1983; Carter and Hansen, 1983).

\section{THE BURGER MODEL}

A unique model to describe both the transient- and steady-state creep process is given by two Zener elements, one of them with very large relaxation times, $\tau_{\epsilon}^{(m)}$. In practice, this is the Burger model, which is formed with a series connection of a Zener element and a dash pot, or equivalently, a series connection of a Kelvin-Voigt element and a Maxwell element (e.g., Klausner, 1991). The response of the Burger model is instantaneous elasticity, delayed elasticity (or viscoelasticity), and viscous flow, the latter described by the dash pot. On removal of the perturbation, the instantaneous and delayed elasticity are recovered, and a viscous flow remains.

The creep function corresponding to $(\mu+3 k)^{-1}$ cannot be solved analytically because the decomposition in partial fractions involves the solution of a fourth-order polynomial to find the relaxation times. The problem has an analytical solution if the shear or bulk moduli are frequency independent. Because the viscoelastic flow mainly results from shear deformations, we assume that the bulk modulus is constant. Figure 1 shows the Burger model for the shear modulus. It is easy to show that the creep function and shear modulus, related by equation 47 , are given by the following equations:

$\chi_{2}(t)=\left\{\frac{1}{\mu_{2}}+\frac{t}{\eta_{2}}+\frac{1}{\mu_{1}}\left[1-\exp \left(-t / \tau_{B}\right)\right]\right\} H(t), \quad \tau_{B}=\frac{\eta_{1}}{\mu_{1}}$

and 


$$
\frac{1}{\mu(\omega)}=\frac{1}{\mu_{2}}+\frac{1}{i \omega \eta_{2}}+\frac{1}{\mu_{1}+i \omega \eta_{1}} .
$$

Furthermore, we obtain

$$
\frac{1}{\mu(\omega)+3 k}=\frac{1}{\mu_{2}+3 k}+\frac{\omega_{1}}{b_{1}\left(i \omega+\omega_{1}\right)}+\frac{\omega_{2}}{b_{2}\left(i \omega+\omega_{2}\right)}
$$

where

$$
\begin{aligned}
\omega_{1} & =\frac{b-\sqrt{b^{2}-4 a c}}{2 a}, \quad \omega_{2}=\frac{b+\sqrt{b^{2}-4 a c}}{2 a}, \\
a & =\eta_{1} \eta_{2}\left(\mu_{2}+3 k\right), \quad b=\mu_{1} \mu_{2} \eta_{2}+3 k d, \quad c=3 k \mu_{1} \mu_{2}, \\
d & =\eta_{2}\left(\mu_{1}+\mu_{2}\right)+\mu_{2} \eta_{1}, \\
b_{1} & =\frac{a \omega_{1}\left(\omega_{2}-\omega_{1}\right)}{\mu_{1} \mu_{2}+\eta_{1} \eta_{2} \omega_{1}^{2}-\omega_{1} d}
\end{aligned}
$$

and

$$
b_{2}=-\frac{a \omega_{2}\left(\omega_{2}-\omega_{1}\right)}{\mu_{1} \mu_{2}+\eta_{1} \eta_{2} \omega_{2}^{2}-\omega_{2} d} .
$$

Equation 102 is similar to 45 , whose creep function is given by equation 50. In this case, we have

$$
\begin{aligned}
\chi(t)= & \left\{\frac{1}{\mu_{2}+3 k}+\frac{1}{b_{1}}\left[1-\exp \left(-\omega_{1} t\right)\right]\right. \\
& \left.+\frac{1}{b_{2}}\left[1-\exp \left(-\omega_{2} t\right)\right]\right\} H(t) .
\end{aligned}
$$

As mentioned above, the Burger model can also be described as a series connection of a Zener element and a dash pot, in this case the dash pot of viscosity $\eta_{2}$. In terms of the Zener parameters, we have

$$
\begin{aligned}
& \tau_{B}=\tau_{\epsilon}^{(2)}, \mu_{1}=\mu_{0}\left(1-\frac{\tau_{\sigma}^{(2)}}{\tau_{\epsilon}^{(2)}}\right)^{-1}, \\
& \mu_{2}=\mu_{0}\left(\frac{\tau_{\epsilon}^{(2)}}{\tau_{\sigma}^{(2)}}\right), \quad \eta_{1}=\mu_{0} \tau_{\epsilon}^{(2)}\left(1-\frac{\tau_{\sigma}^{(2)}}{\tau_{\epsilon}^{(2)}}\right)^{-1} .
\end{aligned}
$$

The strain-stress relations have the form of equation 53, and the strain-displacement relations are given by equations 54 . Using the relative-stress components 41 , we obtain the displacements 58 and 59, where $\chi_{2}$ and $\chi$ are given by equations 100 and 104, respectively.

The major and minor semiaxes vary with time as in equation 71 , with $\chi_{2}$ and $\chi$ given by equations 100 and 104, respectively. The wall-closure time $t_{c}$ is obtained for $a\left(t_{c}\right)=0$, i.e., for

$$
\frac{1}{2}\left(\sigma_{H}+p\right) \chi_{2}\left(t_{c}\right)+\frac{3}{2}\left(\sigma_{H}-\sigma_{h}\right) \chi\left(t_{c}\right)=1 .
$$

The optimal mud weight should satisfy equation 64 with $\sigma_{z z}$ given by equation 62 . The relaxed $(\omega=0)$ and unrelaxed $(\omega=\infty)$ values of the Poisson ratio are $1 / 2$ and $\left(3 k-2 \mu_{2}\right) /\left(3 k+\mu_{2}\right) / 2$, respectively. At the relaxed state, it is $\sigma_{z z}=\sigma_{v}+\sigma_{H}-\sigma_{h}$.

\section{EXAMPLES}

To illustrate the theory, we first compute hole-closure times, and the radius and radial displacements of the borehole wall as a function of time for typical material properties and in-situ stress and temperature conditions. The last example considers a model where a young marine-sediment column (e.g., Gulf of Mexico) has been intruded by a thick homogeneous layer of salt below $2 \mathrm{~km}$ depth. We consider the Louann Salt formation in the Gulf of Mexico (Infante and Chenevert, 1986). Cores of Louann salt, collected from an approximate 4-km depth, have been tested in a triaxial compression cell with the entire surface of the core subject to a fairly constant stress of approximately $91 \mathrm{MPa}$ (the overburden). This stress was applied to core ends by a moveable piston and to the side of the core by hydraulic pressure. The tests were performed for different deviatoric stress by varying the hydraulic pressures at levels below $91 \mathrm{MPa}$. The axial deformation was determined as a function of time for a period of one hour for a range of temperatures.

Figure 3 shows the (a) octahedral stress as a function of effective strain for a range of temperatures, and (b) the elastic and plastic limits as a function of temperature examples for Louann salt as obtained by Infante and Chenevert (1986). Good fits of their data of the octahedral elastic and plastic limits are given by

$$
\tau_{o e}(\mathrm{MPa})=11.4-4.3(T / 100)
$$

and

$$
\tau_{o p}(\mathrm{MPa})=14.0-4.6(T / 100),
$$

where $T$ is given in degrees Celsius.

The steady-state behavior increases with temperature. The steady-state region, where rock salt flows, lies between the two limits. Failure occurs after the plastic limit. The onset of the steady-state behavior decreases with temperature. For low temperatures $\left(24^{\circ} \mathrm{C}\right)$ the Louann salt has linear elastic behavior up to an octahedral stress of about $10 \mathrm{MPa}$. For higher levels of stress the material deforms at a steady-state strain rate and behaves like a viscous fluid at the plastic limit. Typically, for deeper salt deposits exposed to high temperatures $\left(177^{\circ} \mathrm{C}\right)$, the octahedral stress-limits are confined at much lower levels (4-5 MPa). The plot of $\tau_{o e}$ and $\tau_{o p}$ in Figure 3b, with the fitting equations 107 and 108, provide a guide to estimate such stress values at various temperatures.

The physical properties of rocks, including the elastic and plastic limits of rock salt, vary with mean stress as well as with temperature (e.g., Cristescu and Hunsche, 1998). In particular, at low confining pressure (e.g., shallow wells and salt mines), the sensitivity to pressure may be significant. In oil exploration wells, however, which are normally deep and with high confining pressure, the sensitivity to pressure variation is small and thus can be neglected. 
Let us consider first the elastic model. For an overburden average density of $\bar{\rho}=2.4 \mathrm{~g} / \mathrm{cm}^{3}$ and $z=3 \mathrm{~km}$, the vertical stress is $\sigma_{v}=\bar{\rho} g z=71 \mathrm{MPa}$, where $z$ is the depth. The hydrostatic pressure is $p_{H}=\rho_{w} g z=-29 \mathrm{MPa}$, where $\rho_{w}=1 \mathrm{~g} / \mathrm{cm}^{3}$. Assuming $\sigma_{h}$ $=\sigma_{H}=\sigma_{v}$, the borehole pressure to maintain the elastic behavior should satisfy $61 \mathrm{MPa} \leq-p \leq 80 \mathrm{MPa}$, according to equation 30.

Next, we assume nonuniform horizontal loading with $\sigma_{h}$ $=0.8 \sigma_{H}$ and $\sigma_{H}=\sigma_{v}$. The temperature is a function of depth through the geothermal gradient $G$ as $T=z G$. If $G=30^{\circ} \mathrm{C} / \mathrm{km}$, we have $T=90{ }^{\circ} \mathrm{C}, \tau_{o e}=7.5 \mathrm{MPa}$ and $\tau_{o p}=9.9 \mathrm{MPa}$. Because $\tau_{o}$ given by equation 75 is $5.8 \mathrm{MPa}$, i.e., less than $\tau_{o e}$, we use the Zener model to describe the deformation. Reports on the material properties of rock salt for oil-well stability problems are scarce. Studies have generally been performed for underground shallow cavities and salt diapirism (diapir: a relatively mobile mass that intrudes into preexisting rocks). Values of $\mu_{\infty}$ versus temperature are taken from Frost and Ashby (1982):

$$
\mu_{\infty}(\mathrm{GPa})=15\left[1-0.73\left(\frac{T\left({ }^{\circ} \mathrm{C}\right)-27}{1070}\right)\right]
$$

If the relaxed Young's modulus for Louann Salt is assumed to be $Y_{0}=0.3 \mathrm{GPa}$, and $\nu_{\infty}=\nu_{0}=0.25$ during the creep process (a Pois-
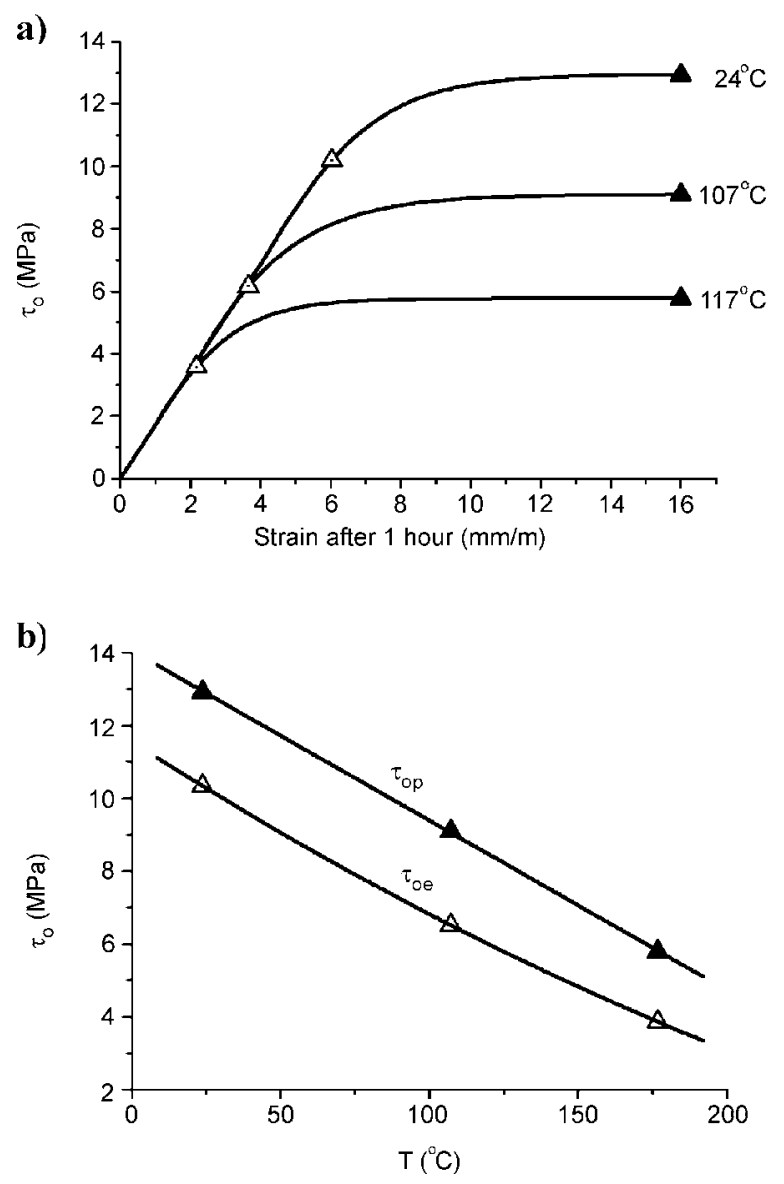

Figure 3. (a) Octahedral stress as a function of octahedral strain for a range of temperatures and elastic (empty triangles) and plastic (solid triangles) limits as a function of (b) temperature for Louann salt (data from Infante and Chenevert, 1986). son solid for which $k=5 \mu / 3)$, use of the preceding equation and the relations: $k=Y /(3-6 \nu), \mu=Y /(2+2 \nu)$, and $Y$ $=9 k \mu /(3 k+\mu)$ yields $\mu_{\infty}=14 \mathrm{GPa}, \quad k_{\infty}=24 \mathrm{GPa}, \quad Y_{\infty}=36$ $\mathrm{GPa}, \mu_{0}=0.08 \mathrm{GPa}$, and $k_{0}=0.13 \mathrm{GPa}$. The value of $k_{\infty}$ before creep is close to 24.8 GPa reported by Mavko et al., (1998).

Urai et al., (1987) have deduced from microstructural studies that in most salt bodies the differential stress is small. We assume here that $\sigma_{h}=0.9 \sigma_{H}$. This assumption and equation 64 give the condition $65 \mathrm{MPa} \leq-p \leq 83 \mathrm{MPa}$. In this case, $\tau_{o e}=\tau_{o r}$, because $\nu_{\infty}=\nu_{0}$ and $\sigma_{z z}$ remains constant during the creep process (see equation 62). The unrelaxed $(t=0)$ and relaxed $(t=\infty)$ minor and major semiaxes of the borehole cross section for the hydrostatic borehole pressure $p_{H}=-29 \mathrm{MPa}$ are $a(0) / r_{0}=0.998$, $b(0) / r_{0}=0.999$ and $a(\infty) / r_{0}=0.74, b(\infty) / r_{0}=0.79$, respectively. If the borehole pressure is $100 \mathrm{MPa}, a(0) / r_{0}=1.0009, b(0) / r_{0}$ $=1.0013$, and $a(\infty) / r_{0}=1.18, b(\infty) / r_{0}=1.23$, then the borehole expands. In the case of an empty borehole $(p=0), a(0) / r_{0}$ $=0.997, b(0) / r_{0}=0.998$ and $a(\infty) / r_{0}=0.55, b(\infty) / r_{0}=0.61$, then the borehole cross section decreases.

Let us assume now that the depth is $z=4 \mathrm{~km}$. The vertical stress is $\sigma_{v}=\bar{\rho} g z=94 \mathrm{MPa}$, and we assume nonuniform horizontal loading with $\sigma_{h}=0.8 \sigma_{H}$ and $\sigma_{H}=\sigma_{v}$. If $G=30^{\circ} \mathrm{C} / \mathrm{km}$, we have $T=120{ }^{\circ} \mathrm{C}, \tau_{o e}=6.2 \mathrm{MPa}$ and $\tau_{o p}=8.5 \mathrm{MPa}$. Because $\tau_{o}$, given by equation 75 is $7.7 \mathrm{MPa}$, i.e., greater than $\tau_{o e}$, we use the Maxwell model to describe the deformation. In this case, $\mu_{\infty}$ $=14 \mathrm{GPa}$, and $k_{\infty}=23 \mathrm{GPa}$. We take $A_{k}=0.462(\mathrm{MPa})^{-n_{k}} \mathrm{~s}^{-1}$, $A_{\mu}=0.462(\mathrm{MPa})^{-n} \mu^{-1}, E_{k}=27 \mathrm{kcal} / \mathrm{mol}, E_{\mu}=23.3 \mathrm{kcal} / \mathrm{mol}$, and $n_{k}=n_{\mu}=5.5$, which correspond to polycrystalline halite (rock salt). The values for the shear deformation have been taken from Parrish and Gangi (1981), and the dilatational deformations have been assumed to have large activation energy and consequently very-high viscosity. Since $R=1.9872 \mathrm{cal} / \mathrm{mol} /{ }^{\circ} \mathrm{K}$, we obtain $\dot{e}_{k}$ $=1.2 \times 10^{-11} \mathrm{~s}^{-1}, \quad \dot{e}_{\mu}=1.4 \times 10^{-9} \mathrm{~s}^{-1}, \quad \eta_{k}=3.2 \times 10^{17} \mathrm{~Pa} \cdot \mathrm{s}$, and $\eta_{\mu}=2.8 \times 10^{15} \mathrm{~Pa} \cdot \mathrm{s}$. With these values, the relaxed Poisson ratio $\nu(\omega=0)=\chi_{\nu}(t=\infty)$ is nearly 0.5 (see equation 90). If $\sigma_{h}$ $=0.9 \sigma_{H}$, the condition of equation 64 for the optimal mud weight gives $96 \mathrm{MPa} \leq-p \leq 111 \mathrm{MPa}$ (unrelaxed case) and $98 \mathrm{MPa}$ $\leq-p \leq 109 \mathrm{MPa}$ (relaxed case).

The hole-closure time $t_{c}$ depends on the value of the borehole pressure $p$ according to equation 94 . If $\sigma_{H}=\sigma_{h}=\sigma_{v}=94 \mathrm{GPa}$, the closure time is given by equation 96 , which gives $1.9 \mathrm{yr}$ and $19 \mathrm{yr}$ for zero borehole pressure (empty hole) and $p=-0.9 \sigma_{h}$, respectively. The borehole radius is constant and equal to $r_{0}$ if $p=-\sigma_{h}$. If the activation energies are $E_{k}=27 \mathrm{kcal} / \mathrm{mol}$ and $E_{\mu}=15 \mathrm{kcal} / \mathrm{mol}$, we have $\dot{e}_{k}=1.2 \times 10^{-11} \mathrm{~s}^{-1}, \quad \dot{e}_{\mu}=5.65$ $\times 10^{-5} \mathrm{~s}^{-1}, \quad \eta_{k}=3.2 \times 10^{17} \mathrm{Pas}$, and $\eta_{\mu}=6.8 \times 10^{10} \mathrm{Pas}$. In this case, the closure times are $4 \mathrm{~h}$ and $24 \mathrm{~h}$ for an empty hole and $p=-0.9 \sigma_{h}$, respectively.

Figure 4 shows the normalized minor and major semiaxis (equation 71) as a function of time for $p=-0.9 \sigma_{h}$ (a) and the closure time as a function of mud pressure $p$ (b). The dashed lines correspond to the elliptical cross section, i.e., when $\sigma_{h}=0.9 \sigma_{H}$ and the solid line is the case $\sigma_{h}=\sigma_{H}$. The upper dashed line corresponds to the major semiaxis. The calculations to obtain Figure $4 \mathrm{~b}$ simplify, since the relaxation frequency $\omega_{1}=0.17 \mathrm{l} / \mathrm{s}$ and $\exp \left(-\omega_{1} t\right) \approx 0$ in equation 84 .

Let us now consider the Burger model with $k=23 \mathrm{GPa}, \mu_{0}$ $=0.08 \mathrm{GPa}$ and $\mu_{\infty}=\mu_{2}=\mu_{0} \tau_{\epsilon}^{(2)} / \tau_{\sigma}^{(2)}=14 \mathrm{GPa}$. If $\sigma_{h}=0.9 \sigma_{H}$, the condition of equation 64 for the optimal mud weight gives nearly the same values reported before for the Maxwell model. To calcu- 
late closure times, we consider $\tau_{B}=\tau_{\epsilon}^{(2)}=5 \mathrm{~h}$ and $\eta_{2}=6.8$ $\times 10^{10} \mathrm{Pas}$. Then, from equation 106 and taking $\sigma_{h}=0.9 \sigma_{H}$ $=0.9 \sigma_{v}$, the closure times are $24 \mathrm{~min}$ and $4 \mathrm{~h}$ for an empty hole and $p=-0.9 \sigma_{H}$, respectively.

The normalized radial displacement at $r=r_{0}$ is obtained from equation 58 :

$\frac{u_{r}}{r_{0}}=\frac{1}{4} \chi_{2}\left(\sigma_{H}+\sigma_{h}+2 p\right)+\frac{1}{2}\left(\sigma_{H}-\sigma_{h}\right)\left(\frac{1}{2} \chi_{2}+3 \chi\right) \cos 2 \theta$.

Figure 5 shows the radial displacement as a function of the azimuthal angle $\theta$ and three different times. The horizontal tectonic stresses are $\sigma_{H}=\sigma_{v}$ and $\sigma_{h}=0.9 \sigma_{H}$, and the mud pressure is $p=$ $-0.9 \sigma_{H}$.

Next, we consider a realistic example, with $E_{k}=27 \mathrm{kcal} / \mathrm{mol}$ and $E_{\mu}=20 \mathrm{kcal} / \mathrm{mol}$. Typical values of the activation energies can be found in Parrish and Gangi (1981). The octahedral-stress limits are obtained from equations 107 and 108. Figure 6a shows a model where a young marine-sediment column (e.g., Gulf of Mexico) has been intruded by a 3-km-thick homogeneous layer of salt at levels below $2-\mathrm{km}$ depth. The salt is characterized by a neg-
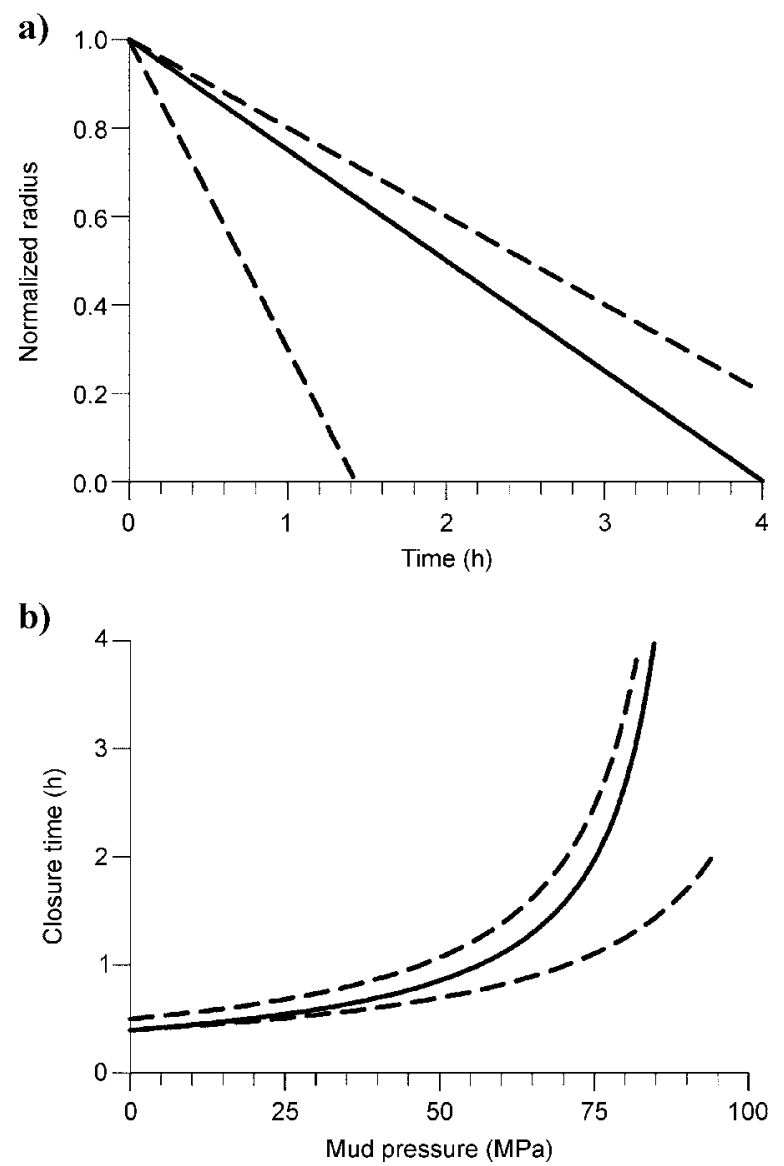

Figure 4. (a) Minor and major semiaxis, $a(t) / r_{0}$ and $b(t) / r_{0}$ (dashed lines, equation 71), versus time for $p=-0.9 \sigma_{h}$ and (b) closure time versus mud pressure $p$. We have assumed $\sigma_{H}=\sigma_{v}$. The dashed lines correspond to the elliptical cross section, i.e., when $\sigma_{h}=0.9 \sigma_{H}$, and the solid line is the case $\sigma_{h}=\sigma_{H}$. The upper dashed line corresponds to the major semiaxis. The depth is $4 \mathrm{~km}$, and the medium is described by the Maxwell model. ligible small porosity $\phi$ and a density of $2.1 \mathrm{~g} / \mathrm{cm}^{3}$. Assuming a brine pore fill $\left(1.03 \mathrm{~g} / \mathrm{cm}^{3}\right)$, we have computed the bulk density $\rho$ and the corresponding overburden stress $\sigma_{v}$ using common procedures (e.g., Carcione et al., 2003). To comply with the practice in drilling engineering, we express the overburden stress and mud pressure by their equivalent mud weights, or the equivalent densities of the mud needed to balance pressure at given depths. Using a geothermal gradient of $30^{\circ} \mathrm{C} / \mathrm{km}$, we have established the elastic and plastic limits $\tau_{o e}$ and $\tau_{o p}$, respectively, within the salt layer.

In the first experiment (I) (shown in Figure 6b), we assume an isotropic stress distribution where $\sigma_{H}=\sigma_{h}=\sigma_{v}$, and the resulting limits $p_{1}(I)$ and $p_{2}(I)$ vary symmetrically versus depth with respect to $\sigma_{v}$ and with $p_{1}(I)$ well below the overburden pressure. In the second experiment (II), we introduce a nonuniform loading with $\sigma_{h}=0.8 \sigma_{H}$ and $\sigma_{H}=1.1 \sigma_{v}$. The latter values are consistent with values of stress anisotropy at prospective depths provided by Hegret (1987) and Arjang (1989) for the Canadian Shield, and in agreement with the global picture presented by Engelder (1993, p.91). In this case, the minimum mud weight $p_{1}(I I)$ exceeds the overburden stress in most of the salt section, except below the depth where the initial deviatoric stress $\tau_{o}$ (i.e., equation 6) approach the plastic limit $\tau_{o p}$. The elastic-to-plastic transition implies a slow creep that is sufficient to relieve any significant stress differences over geologic time. Below this critical level, the stress patterns within the salt will tend towards isotropy; hence, elasticity will be recovered (see Figure $3 \mathrm{a}$ ), and the elastic condition in equation 64 for the borehole pressure applies.

Drilling in salt with a borehole pressure at the overburden gradient, even with heavier mud, is a common experience in the Zagros Fold Belt (Iran) - one of the motivations for the present study. Here, a thick layer of salt in the Gachsaran Formation (e.g., see Bahroudi and Koyi, 2004) forms the cap rock covering the major hydrocarbon reservoirs in the region. A lateral shortening in Zagros of approximately $10 \mathrm{~mm} / \mathrm{y}$, inferred from GPS measurements by Tartar et al. (2002), is associated with a strong nonuniform loading (Bird, 1978). Hence, experiment II in Figure 6b portrays a realistic situation.

Now, suppose that the well has been drilled using the minimum mud pressure $p=-p_{1}$ as in case II of Figure 6b. Then, we follow the development in time of the borehole geometry and eventually further adjust the mud pressure to optimize the hole stability as shown in Figure 7 at 10, 50, and 100 hs after drilling. The Maxwell viscoelastic model is applied with the viscosities given by equation

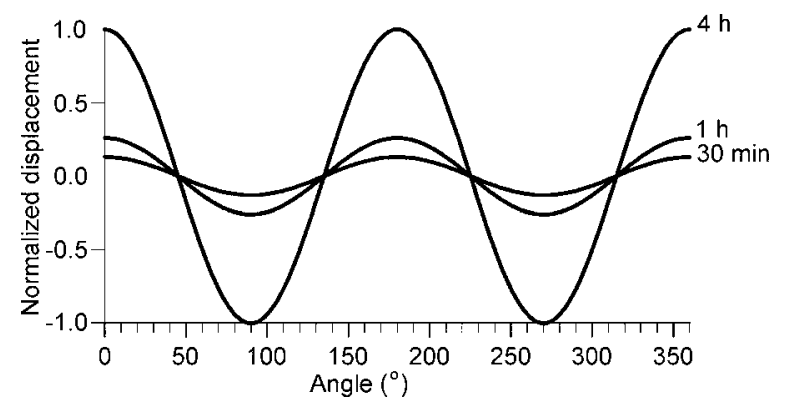

Figure 5. Normalized radial displacement $\left(u_{r} / r_{0}\right)$ versus azimuthal angle at three different times. The depth is $4 \mathrm{~km}$, and medium is described by the Burgers model. The horizontal tectonic stresses are $\sigma_{H}=\sigma_{v}$ and $\sigma_{h}=0.9 \sigma_{H}$, and the mud pressure is $p=-0.9 \sigma_{H}$. 
97, but with an upper bound $\tau_{o} \leq \tau_{o p}$ (see equation 108) applied to equations 98 and 99.

For mud pressure $p=-p_{1}$, the borehole through the top salt section remains stable, whereas the lower part expands by about $10 \%$ after $100 \mathrm{hs}$ at the elastic-plastic transition boundary $(4.36 \mathrm{~km})$. Below this critical level, the borehole contracts by about $5 \%$ in $100 \mathrm{hs}$. Obviously, a mud pressure of $p=-p_{1}$ fails to stabilize the borehole, and we adjust the mud weight to improve the situation as shown in Figure 8 with $p=-1.05 p_{1}$. Now, with slightly heavier mud, the changes in the borehole are less than $1 \%$ after 100 hs and only about $2 \%$ after 1000 hs, which normally should be sufficient time to drill and set the casing. Setting casing at the transition level would be required before drilling with reduced mud weights in the lower section.

The anisotropic-stress state (i.e., when deviatoric stress exists) would require mud weight in excess of overburden (i.e., borehole

a)

$$
\sigma_{v}, \rho\left(\mathrm{g} / \mathrm{cm}^{3}\right)
$$

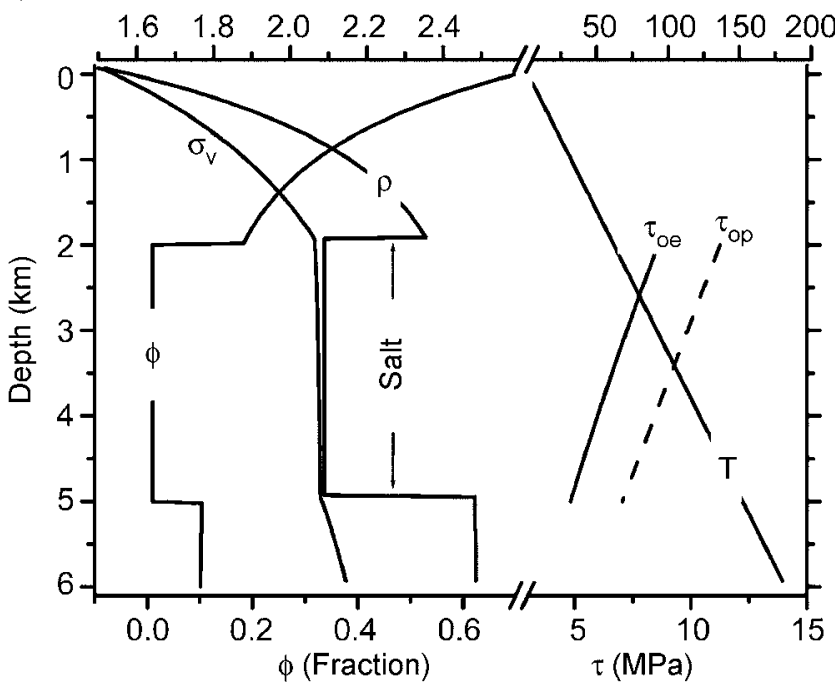

b)

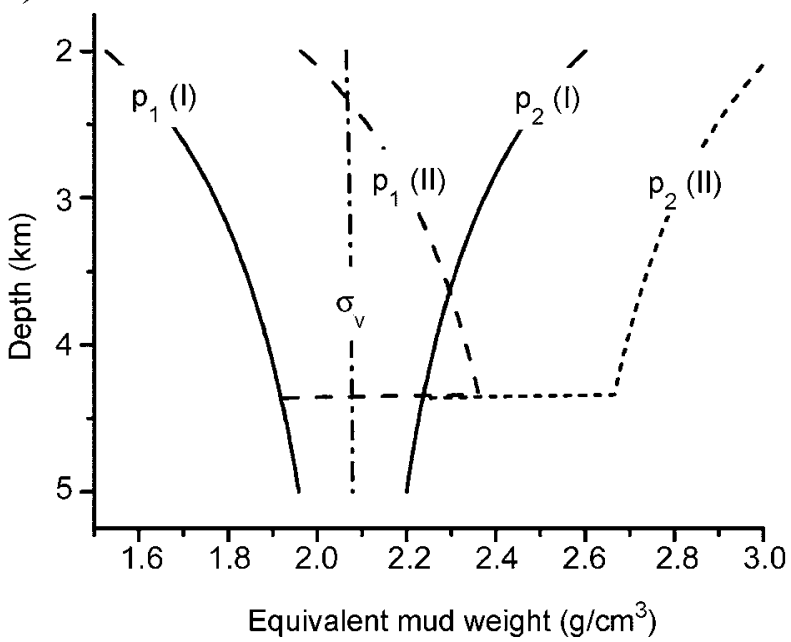

Figure 6. (a) A realistic (e.g., Gulf of Mexico) sediment-compaction model containing a buried salt layer with negligible porosity $\phi$ and density $\rho$, and the corresponding mud pressure $p_{1}$ and $p_{2}$ (in equivalent mud weight) at the elastic limits (equations 65 and 66) for two models of the initial stress; I (isotropic): $\sigma_{H}=\sigma_{h}=\sigma_{v}$; II (anisotropic): $\sigma_{h}=0.8 \sigma_{H} ;$ and $\sigma_{H}=1.1 \sigma_{v}$ pressures greater than the overburden pressure) only if the horizontal principal stresses (one or both) exceed the overburden stress. If the horizontal principal stresses are both less than the overburden (vertical principal) stress, then the borehole pressure need not exceed the overburden stress for stability. Obviously, if the horizontal (isotropic) principal stress exceeds the overburden stress, borehole pressure greater than the overburden will be needed to prevent intrusion of the salt. However, the formation would be hydrofractured; i.e., borehole fluid would be injected into the formation. However, fracture pressure in salt has proved to be much higher than in nonsalt sections at a comparable depth when drilling in the Gulf of Mexico (Barker and Meeks, 2003).

To our knowledge, the only published caliper measurements of shrinkage and expansion of a real well bore in a salt layer (probably Louann salt), as a function of time for three different mud weights, were made by Kim (1988). His results agree qualitatively with our theory; i.e., for highly underbalanced mud $(14.3 \mathrm{lb} / \mathrm{gal})$, the hole shrinkage (18 inches at $17,700 \mathrm{ft})$ was significant $(2.3$ inches in 12 hours), and by increasing the mud weight (to 15.3 $\mathrm{lb} / \mathrm{gal})$ the shrinkage was reduced (0.6 inches in 12 hours). With
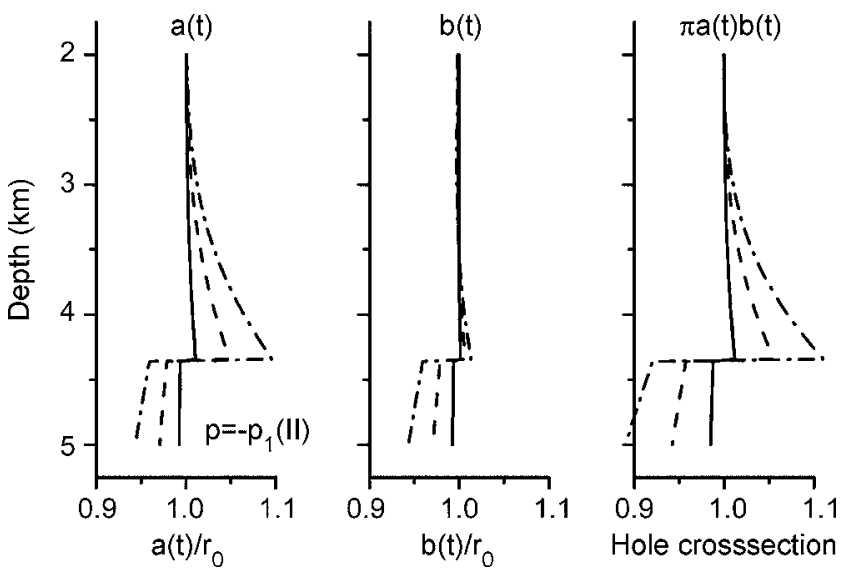

Figure 7. Variation with depth of the minor axis $a(t)$ and major axis $b(t)$ (see equation 71) and normalized cross-section area after 10 , 50, and 100 hs. The model corresponds to case II in Figure 6b using mud pressure $p=-p_{1}(I I)$.
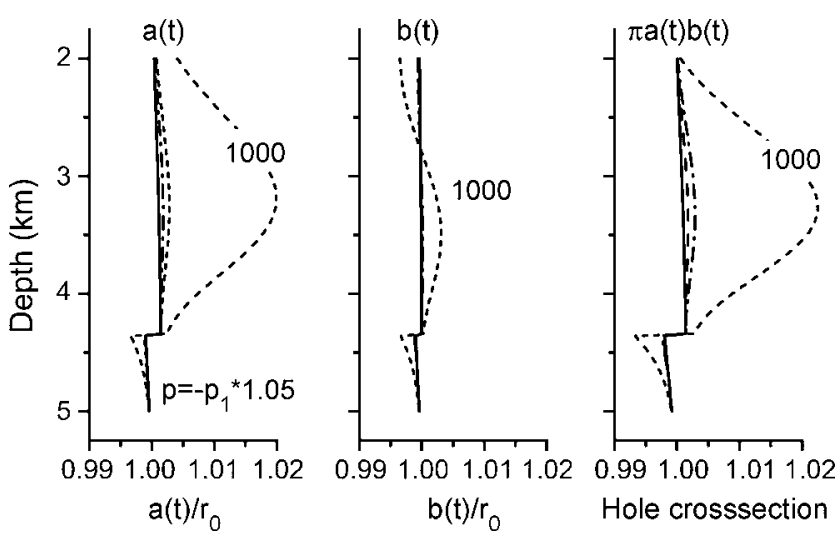

Figure 8. Same variations as in Figure 7 but with a small change in mud pressure to $p=-1.05 p_{1}(I I)$ in an attempt to stabilize the hole. Note that after $1000 \mathrm{hs}$, the change in the hole diameter is less than $2 \%$. 
heavier mud weight $(17.3 \mathrm{lb} / \mathrm{gal})$, the caliper indicated stability or a slight expansion of the hole. For operational reasons, each experiment was terminated with only one caliper run after 12 hours. The data provided by Kim (1988) are thus not sufficient for a quantitative comparison with our theory.

The theory developed here can be used also to evaluate hole stability when drilling through shale formations. The analysis would be valid when the shale behaves like rock salt, i.e., when it flows fairly readily, which happens at low effective pressures (for instance, there are shale diapirs in the Gulf of Mexico). See Johnston (1987) for experimental data for shale on viscoelastic and creep properties as a function of pressure and temperature.

\section{CONCLUSION}

We have developed a theoretical approach to analyze the stability of boreholes during and after drilling. We obtain expressions for the shape of the borehole cross section, the borehole-wall closure time, and the optimal mud weight to avoid wall collapse and expansion. The theory describes transient creep by the Zener model and steady-state creep by the Maxwell model or by the Burger model. The Zener model is used under pressure and temperature conditions, where the rock behaves as a viscoelastic solid. The choice depends on the value of the elastic octahedral stress which determines the limit separating transient flow from unrecoverable steady-state flow.

Examples for the Louann Salt formation are given. At 3-km depth, the vertical overburden stress is $71 \mathrm{MPa}$, and the octahedral-stress limit for elastic or viscoelastic transient behavior is 7.5 MPa. Under isotropic tectonic-stress conditions, the theory predicts a range of mud pressure between 61 and $80 \mathrm{MPa}$ to stabilize the borehole. On the other hand, if the maximum horizontal stress is equal to the vertical stress and there is a differential horizontal tectonic stress of $20 \%$, the predicted range is $(65,83) \mathrm{MPa}$. Under these conditions, the minor semiaxis of the hole elliptical cross section decreases by $45 \%$ if the borehole is empty and by $25 \%$ if it is filled with water (a hydrostatic pressure of $-29 \mathrm{MPa}$ ). On the other hand, the semiaxis increases by nearly $20 \%$ if the mud pressure is $-100 \mathrm{MPa}$, i.e., $29 \mathrm{MPa}$ higher than the vertical overburden stress.

At a 4-km depth, the vertical overburden stress is $94 \mathrm{MPa}$, the octahedral stress limit is $6.2 \mathrm{MPa}$, and rock salt shows viscoelastic flow. The Maxwell and Burger models predict a range of mud pressure of $(96,111) \mathrm{MPa}$ and $(98,109) \mathrm{MPa}$ for the unrelaxed (immediately after drilling) and relaxed states, respectively. The holeclosure times depend on the value of the steady-state creep rates and viscosities through the activation energies. Assuming that the shear deformations are dominant, a rock salt viscosity of the order of $10^{15} \mathrm{~Pa}$ s gives a closure time of a few years for an empty hole, while the closure time is $24 \mathrm{~min}$ for a viscosity of nearly $10^{11} \mathrm{~Pa}$.

By adjusting the mud weight, we may keep constant the elliptical cross-section area, and hence the mud volume, but fail to control the hole geometry at the same time. For deeply buried salt exposed to tectonic forces, the in-situ octahedral stress may approach the plastic limit; and thus, the salt will be subject to creep. Since creep over geologic time is likely to relieve any significant stress differences, the elasticity will recover and hole stability below the critical depth is feasible.

In the example presented here, we have defined different regimes based on the value of the octahedral stress. We model steady-state creep effects with the Maxwell or Burger model below a given depth determined by the in-situ temperature conditions. Alternatively, the Maxwell or Burger models can be used over the whole range of octahedral stresses. In this case, the method requires a precise determination of the activation energies as a function of the octahedral stress and temperature.

The preceding predicted values are very sensitive to the anelastic properties of rock salt and the in-situ stress and temperature conditions. A precise evaluation of the borehole stability requires a detailed characterization of rock salt behavior by means of laboratory experiments. The theory has been applied to rock salt but can also be used to evaluate hole stability when drilling through shale formations. In some cases, the inclusion of poroelastic effects and borehole inclination can be important.

\section{ACKNOWLEDGMENTS}

This work was supported by Norsk Hydro a. s. (Bergen). We thank Fabio Cavallini for helping with the mathematical software.

\section{REFERENCES}

Abouleisman, Y., A. H.-D. Cheng, C. Jiang, and J.-C. Roegiers, 1996, Poroviscoelastic analysis of borehole and cylinder problems: Acta mechanica, 119, 199-219.

Abouleisman, Y., and L. Cui, 1998, Poroelastic solutions in transversely isotropic media for wellbores and cylinders: International Journal of Solids and Structures, 35, 4905-4929.

Arjang, B., 1989, Pre-mining stresses at some hard rock mines in the Canadian Shield. Rock mechanics as a guide for efficient utilization of natura resources: Proceedings of the 30th Annual U. S. Symposium of Rock Mechanics, 545-551.

Auld, B. A., 1991, Acoustic fields and waves in solids, vol. 1: Robert E. Krieger, Publishing Company.

Bahroudi, A., and H. A. Koyi, 2004, Tectono-sedimentary framework of the Gachsaran Formation in the Zagros foreland basin: Marine and Petroleum Geology, 21, 1295-1310.

Barker, J. W., and W. R. Meeks, 2003, Estimating fracture gradient in Gulf of Mexico deepwater, shallow massive salt sections: SPE-84552.

Ben-Menahem, A., and S. J. Singh, 1981, Seismic waves and sources: Springer-Verlag.

Bird, P., 1978, Finite element modeling of lithosphere deformation, The Zagros collision orogeny: Tectonophysics, 50, 307-336.

Bradley, W. B., 1978, Borehole failure near salt domes: SPE-7503.

Carcione, J. M., 2001, Wave fields in real media: Theory and numerical simulation of wave propagation in anisotropic, anelastic and porous media: Pergamon Press.

Carcione, J. M., H. B. Helle, N. H. Pham, and T. Toverud, 2003, Pore pressure estimation in reservoir rocks from seismic reflection data: Geophysics, 68, 1569-1579.

Carter, N. L., and F. D. Hansen, 1983, Creep of rock salt: Tectonophysics, 92, 275-333.

Charlez, P. A., 1991, Rock mechanics, theoretical fundamentals, vol. 1: Editions Technip.

Cristescu, N., 1989, Rock rheology: Kluwer Academic Publishers.

Cristescu, N. D., and U. Hunsche, 1998, Time effects in rock mechanics: John Wiley \& Sons.

Cui, L., A. H. D. Cheng, and Y. Abouleisman, 1997, Poroelastic solution for an inclined borehole: Transactions of the ASME, 64, 32-38.

Ekbote, S., and Y. Abouleisman, 2003, Poromechanics stability analysis for inclined wellbores under non-isothermal conditions in chemically active formations: 16th ASCE Engineering Mechanics Conference.

Engelder, T., 1993, Stress regimes in the lithosphere: Princeton University Press.

Frost, H. J., and M. F. Ashby, 1982, Deformation mechanism maps: The plasticity and creep of metals and ceramics: Pergamon Press.

Gangi, A. F., 1981, A constitutive equation for one-dimensional transient and steady-state flow of solids: Mechanical behavior of crustal rocks, Geophysical Monograph 24: AGU, 275-285.

, 1983, Transient and steady-state deformation of synthetic rock salt: Tectonophysics, 91, 137-156.

Ghassemi, A., and A. Diek, 2001, Effects of ion transfer on stress and pore pressure distributions around a borehole in shale: Proceedings of the 38th Annual U. S. Symposium of Rock Mechanics. 
Hegret, G., 1987, Stress assumption for underground excavation in the Canadian Shield: Internationa Journal of Rock Mechanics and Mining Sciences and Geomechanics Abstracts, 24, 95-97.

Infante, E. F., and M. E. Chenevert, 1986, Stability of boreholes drilled through salt formations displaying plastic behavior: SPE-1553.

Jaeger, J. C., and N. G. W. Cook, 1969, Fundamental of rock mechanics: Chapman and Hall Ltd. and Science Paperbacks.

Johnston, D. H., 1987, Physical properties of shale at temperature and pressure: Geophysics, 52, 1391-1401.

Karasudhi, P., 1991, Foundations of solid mechanics: Kluwer Academic Publishers.

Kim, C. M., 1988, Field measurements of borehole closure across salt formation: Implementing to well cementing, 63rd Annual Fall Technical Conference and Exhibition of the Society of Petroleum Engineering, 2-5.

Kirsch, G., 1898, Die theorie der elastizität und die bedürfnisse der festigkeitslehre: Zeitschrift Verein Deutscher Ingenieure, 42, 797-807.

Klausner, Y., 1991, Fundamentals of continuum mechanics of soils: Springer-Verlag.

Ladanyi, B., and D. E. Gill, 1983, In-situ determination of creep properties of rock salt: 5th International Congress on Rock Mechanics, A219-A225.

Leblanc, L., 1994a, Part I: Drilling, completion, workover challenges in subsalt formations: Offshore, 21-22.

-, 1994b, Part II: Drilling, completion, workover challenges in subsalt formations: Offshore, 42-44.

-, 1994c, Part III: Drilling, completion, workover challenges in subsalt formations: Offshore, 38-40.

Mavko, G., T. Mukerji, and J. Dvorkin, 1998, The rock physics handbook: tools for seismic analysis in porous media: Cambridge University Press Pande, G. N., G. Beer, and J. R. Williams, 1990, Numerical methods in rock mechanics: John Wiley \& Sons.

Parrish, D. K., and A. F. Gangi, 1981, A nonlinear least-squares technique for determining multiple-mechanism, high-temperature-creep flow laws: Geophysical Monograph 24: AGU, 287-298.

Raymer, D. G., A. Tommasi, and J. M. Kendall, 2000, Predicting the seismic implications of salt anisotropy using numerical simulations of halite deformation: Geophysics, 65, 1272-1280.

Roegiers, J. C., 2002, Well modeling: An overview: Oil and Gas Science and Technology 569-577.

Serata, S., and E. F. Gloyna, 1960, Principles of structural stability of underground salt cavities: Journal of Geophysical Research, 65, 29792987.

Sun, Z., R. J. Brown, D. C. Lawton, and Z. Wang, 1991, Seismic anisotropy and salt detection: a physical modeling study: 61st Annual International Meeting, SEG, Expanded Abstracts, 713-716.

Tartar, M., D. Hatzfeld, J. Martinod, A. Walpersdorf, M. Ghafori-Ashtiany, and J. Chéry, 2002, The present-day deformation of the central Zagros from GPS measurements: Geophysics Research Letters, 29, 19.

Urai, J. L., C. J. Spiers, C. J. Peach, R. C. M. W. Franssen, and J. L. Liezenberg, 1987, Deformation mechanisms operating in naturally deformed halite rocks as deduced from microstructural investigations: Geologie en Mijnbouw, 66, 165-176.

Willson, S. M., A. F. Fossum, and J. T. Fredrich, 2003, Assessment of salt loading on well casings: SPE-81820.

Zener, C., 1948, Elasticity and anelasticity of metals: University of Chicago Press. 